\title{
Leptogenesis from loop effects in curved spacetime
}

\author{
Jamie I. McDonald and Graham M. Shore \\ Department of Physics, Swansea University, \\ Singleton Park, Swansea, SA2 8PP U.K. \\ E-mail: pymcdonald@swansea.ac.uk, g.m.shore@swansea.ac.uk
}

AbSTRACT: We describe a new mechanism - radiatively-induced gravitational leptogenesis - for generating the matter-antimatter asymmetry of the Universe. We show how quantum loop effects in $\mathrm{C}$ and $\mathrm{CP}$ violating theories cause matter and antimatter to propagate differently in the presence of gravity, and prove this is forbidden in flat space by CPT and translation symmetry. This generates a curvature-dependent chemical potential for leptons, allowing a matter-antimatter asymmetry to be generated in thermal equilibrium in the early Universe. The time-dependent dynamics necessary for leptogenesis is provided by the interaction of the virtual self-energy cloud of the leptons with the expanding curved spacetime background, which violates the strong equivalence principle and allows a distinction between matter and antimatter. We show here how this mechanism is realised in a particular BSM theory, the see-saw model, where the quantum loops involve the heavy sterile neutrinos responsible for light neutrino masses. We demonstrate by explicit computation of the relevant two-loop Feynman diagrams how the size of the radiative corrections relevant for leptogenesis becomes enhanced by increasing the mass hierarchy of the sterile neutrinos, and show how the induced lepton asymmetry may be sufficiently large to play an important rôle in determining the baryon-to-photon ratio of the Universe.

Keywords: Classical Theories of Gravity, CP violation, Neutrino Physics, Effective field theories

ArXiv EPrint: 1512.02238 


\section{Contents}

1 Introduction 1

1.1 The mechanism 2

2 Matter-antimatter propagation in flat and curved spacetime $\quad 6$

$\begin{array}{lll}2.1 & \text { Propagation in translation invariant backgrounds } & 7\end{array}$

$\begin{array}{llr}2.2 & \text { Propagation in curved backgrounds } & 9\end{array}$

3 Effective field theory - integrating out the sterile neutrinos 11

$\begin{array}{lll}3.1 \text { Matching } & 12\end{array}$

4 Feynman diagram calculations $\quad \mathbf{1 5}$

$\begin{array}{lll}4.1 & \text { Sterile neutrino couplings } & 16\end{array}$

4.2 Yukawa couplings 23

4.3 Higgs couplings 24

5 Leptogenesis and baryogenesis $\quad \mathbf{2 4}$

5.1 Lepton asymmetry in a radiation-dominated FRW background 24

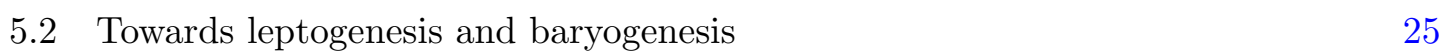

6 Outlook 27

$\begin{array}{ll}\text { A Techniques for evaluating the self-energy diagrams } & 30\end{array}$

\section{Introduction}

Recently, we presented a new mechanism [1] for generating matter-antimatter asymmetry in the Universe by exposing a deeper connection between matter, antimatter and gravity. Our central finding was that at the quantum loop level, matter and antimatter propagate differently in the presence of gravity when $\mathrm{C}$ and $\mathrm{CP}$ are violated - a phenomenon we showed to be forbidden in flat space by translation invariance and CPT. This leads to a difference in the dispersion relations for matter and antimatter, which manifests itself in the form of a chemical potential for baryons or leptons in an effective action, and leads to a mechanism for generating the baryon asymmetry of the Universe without invoking out-of-equilibrium decays of heavy BSM particles. In this paper, we explain these ideas in greater detail and give an extended account of the calculations which underlie them.

This mechanism - radiatively-induced gravitational (baryo) leptogenesis - is very general, and relies only on the breaking of three symmetries: C, CP and spacetime translation invariance. This is realised in a cosmological setting by the Hubble expansion of the Universe, which provides the time-dependent dynamics necessary to produce a matterantimatter asymmetry. Consequently, many flat space BSM theories of baryogenesis and leptogenesis, which by virtue of the Sakharov conditions [2] must violate C and CP, will 
naturally exhibit our mechanism when minimally coupled to gravity, without the need to add new fundamental interactions. Moreover, and remarkably for an intrinsically quantum field theoretic effect in curved spacetime, it is strong enough to generate the observed baryon-to-photon ratio of the Universe.

In the remainder of this section, we introduce the key ideas of our mechanism in the context of leptogenesis, illustrated in the popular 'see-saw' model [3] in which heavy sterile neutrinos are introduced to provide masses for the SM neutrinos. We contrast the way in which leptogenesis arises in this model through the coupling of quantum loops involving virtual sterile neutrinos to the cosmological gravitational field with the conventional mechanism, where the lepton asymmetry arises from the decays of on-shell sterile neutrinos as they fall out of equilibrium as the Universe cools.

After this overview, we describe our approach in detail from first principles, beginning in section 2, where we discuss radiative corrections to matter and antimatter propagation in flat and curved backgrounds. Here, we demonstrate that when $\mathrm{C}$ and $\mathrm{CP}$ are violated, the breaking of translational invariance by gravity leads to a difference in the propagation of matter and antimatter. We also provide a simple proof, at the level of both S-matrix elements and correlators, that CPT and translation invariance prevent this situation in flat space.

In section 3 we study the effective field theory generated by these propagators. We show that when we integrate out the heavy degrees of freedom to construct a low energy effective Lagrangian, distinct matter and antimatter propagation generates a $\mathrm{C}$ and $\mathrm{CP}$ violating operator coupling the lepton current to the derivative of the Ricci scalar. In isotropic cosmologies, this operator leads to a chemical potential between leptons and antileptons, generating a lepton asymmetry driven by the expansion of the Universe. Since $\mathrm{CP}$ violation in the see-saw model arises first at fourth order in the complex coupling between the light and sterile neutrinos, the asymmetry is generated by two-loop Feynman diagrams contributing to the lepton self-energy. The evaluation of the relevant Feynman diagrams is presented in section 4 , where we study in detail how our effect is enhanced by increasing the hierarchy of sterile neutrino masses.

The implications for leptogenesis in the early Universe are discussed briefly in section 5 , where we evaluate the quasi-equilibrium lepton asymmetry in a radiation-dominated FRW universe and show that this may be sufficiently large to play an important rôle in determining the observed baryon-to-photon ratio of the Universe.

\subsection{The mechanism}

Although our mechanism is very general, for clarity we illustrate it in this paper within a particular model - the see-saw model, first proposed as a means of obtaining a baryon asymmetry via leptogenesis by Fukugita and Yanagida [3]. The corresponding minimally coupled Lagrangian is given by

$$
\mathcal{L}=\mathcal{L}_{E W}+\sqrt{-g}\left[\bar{N} i \not D N+\lambda_{i \alpha} \bar{\ell}_{i} \phi N_{\alpha}+\frac{1}{2} \overline{\left(N^{c}\right)} M N+\text { h.c. }\right] .
$$


where $\sqrt{-g}$ is the square root of the metric determinant, and $D$ is the spinor covariant derivative. In this model, $\ell_{i}(i=e, \mu, \tau)$ are the light, left-handed lepton doublets and $\phi$ is the Higgs field ${ }^{1}$ which couples to heavy right-handed sterile neutrinos $N_{\alpha}$ with non-degenerate masses $M_{\alpha}(\alpha=1, \ldots n)$. Crucially, the Yukawa couplings $\lambda_{i \alpha}$ contain irremovable complex phases, providing a source of $\mathrm{C}$ and $\mathrm{CP}$ violation. The first Sakharov condition is realised in two parts: the Yukawa interaction violates lepton number by one unit, allowing the creation of matter-antimatter asymmetry in the lepton sector. This is then converted to a baryon asymmetry of the same magnitude via sphaleron processes $[4,5]$, which are in equilibrium at high temperatures in the early Universe.

We note also at this point that that the Majorana mass term for the heavy neutrinos gives two classes of propagators, charge-violating propagators $S_{\alpha}^{\times}\left(x, x^{\prime}\right)=\left\langle N(x) \overline{N^{c}}\left(x^{\prime}\right)\right\rangle$ and charge-conserving propagators $S_{\alpha}\left(x, x^{\prime}\right)=\left\langle N_{\alpha}(x) \bar{N}_{\alpha}\left(x^{\prime}\right)\right\rangle$, where the $C$ script denotes the Dirac charge conjugate. All diagrams can be expressed in terms of this basis of sterile neutrino propagators. In flat space, translation invariance allows us to write them in momentum space as

$$
S_{\alpha}(p)=\frac{i \not p}{p^{2}-M_{\alpha}^{2}}, \quad S_{\alpha}^{\times}(p)=\frac{i M_{\alpha}}{p^{2}-M_{\alpha}^{2}} .
$$

We now describe how the third Sakharov condition, namely a departure from equilibrium, is satisfied in the traditional sterile neutrino decay scenario, and then how leptogenesis is realised in our own mechanism.

Leptogenesis from heavy neutrino decays. The traditional leptogenesis model neglects gravitational effects in the Lagrangian, with all relevant amplitudes calculated in flat space. In the simplest leptogenesis scenario [3, 6-8], one assumes a thermal initial abundance of sterile neutrinos. ${ }^{2}$ At early times, when the temperature is sufficiently high $\left(T \gtrsim M_{1}\right)$, decays $N_{1} \rightarrow \ell \phi$ and inverse decays $\phi \ell \rightarrow N_{1}$ are in equilibrium and the sterile neutrinos remain thermalised with relativistic number densities. However, as the temperature drops to $T \lesssim M_{1}$, the inverse decays are Boltzmann suppressed by a factor $e^{-M_{1} / T}$ and become inefficient. For a short time, this leaves the sterile neutrinos slightly overabundant compared to their non-relativistic equilibrium distribution, until eventually they decay. This is the out-of-equilibrium process necessary to satisfy the Sakharov condition and allow leptogenesis.

An interference between the tree-level and one-loop decay amplitudes shown in figure 1 gives a difference in the production rates of leptons and antileptons at the time of the heavy decays, as characterised by the well-known quantity [7]

$$
\epsilon_{\alpha}=-\frac{1}{8 \pi} \sum_{\beta \neq \alpha} \frac{\operatorname{Im}\left[\left(\lambda^{\dagger} \lambda\right)_{\alpha \beta}^{2}\right]}{\left(\lambda^{\dagger} \lambda\right)_{\alpha \alpha}}\left[f\left(\frac{M_{\beta}^{2}}{M_{\alpha}^{2}}\right)+g\left(\frac{M_{\beta}^{2}}{M_{\alpha}^{2}}\right)\right],
$$

\footnotetext{
${ }^{1}$ In this notation, the Higgs doublet $\tilde{\phi}$ appearing in the SM lepton sector is related by $\phi^{a}=\epsilon^{a b} \tilde{\phi}^{\dagger b}$.

${ }^{2}$ There are other scenarios [8] for the initial state of the sterile neutrinos, but in all cases, the lepton asymmetry is generated when they depart from their equilibrium distribution.
} 

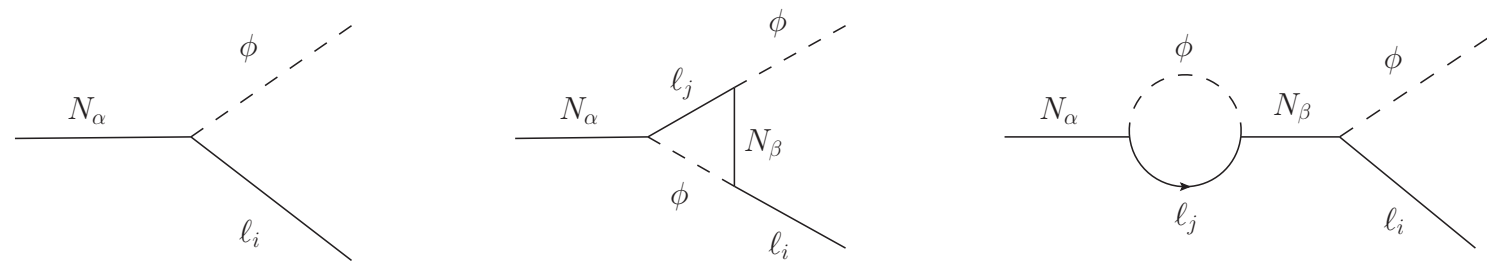

Figure 1. Flat space diagrams contributing to out-of-equilibrium decays. The second and third diagrams give $f(x)$ and $g(x)$ respectively.
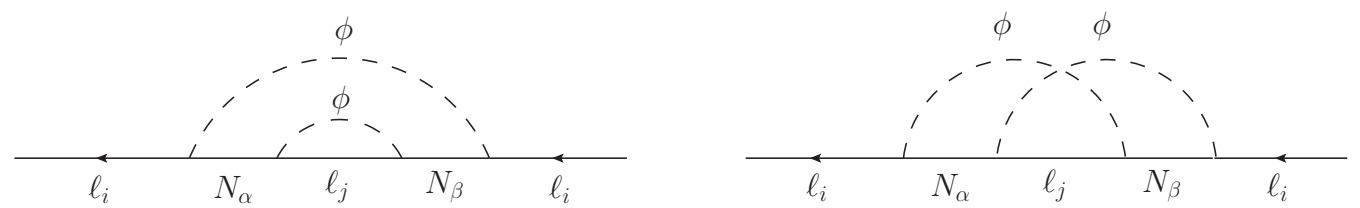

Figure 2. Curved space two-loop self-energies which contribute to radiatively-induced gravitational leptogenesis.

where

$$
\begin{aligned}
& f(x)=\sqrt{x}\left(1-(1+x) \ln \left(\frac{1+x}{x}\right)\right), \\
& g(x)=\frac{\sqrt{x}}{1-x} .
\end{aligned}
$$

In this way, a lepton asymmetry is generated at the point when the sterile neutrinos fall out of equilibrium.

Leptogenesis from radiatively-induced gravitational couplings. In our scenario, the time-dependent dynamics necessary to distinguish the dispersion relations for leptons and antileptons and induce the matter-antimatter asymmetry arises from the expansion of the Universe itself, through the gravitational coupling to the virtual self-energy cloud screening the leptons at the quantum loop level. Here, the heavy sterile neutrinos play an altogether different role. Rather than generating the lepton number asymmetry through their out-of-equilibrium decays, they contribute to our mechanism only as virtual particles mediating the propagation of the light leptons, as shown by the diagrams in figure 2 . The propagators in these self-energy diagrams are the appropriate curved space Green functions (see, e.g., ref. [1]) derived from the minimally-coupled Lagrangian (1.1). Crucially, the introduction of a scale associated with the mass of the virtual particles in the loops allows a direct coupling to the curvature, which violates the strong equivalence principle and allows the leptons and antileptons to propagate differently.

When the heavy sterile neutrinos are integrated out from the Lagrangian (1.1), these self-energy diagrams produce the following $\mathrm{C}$ and $\mathrm{CP}$ violating operator in the low-energy effective action:

$$
\mathcal{L}_{i}=\partial_{\mu} R\left(\bar{\ell}_{i} \gamma^{\mu} \ell_{i}\right) \sum_{\alpha, \beta, j} \frac{\operatorname{Im}\left[\lambda_{\beta i}^{\dagger} \lambda_{i \alpha} \lambda_{\beta j}^{\dagger} \lambda_{j \alpha}\right]}{3 M_{\alpha} M_{\beta}} I_{[\alpha \beta]},
$$


where the loop factor $I_{\alpha \beta}=I\left(M_{\alpha}, M_{\beta}\right)$, which we shall discuss at length in subsequent sections, is a function of the two sterile neutrino masses $M_{\alpha}$ and $M_{\beta}$ in the loop. In a homogeneous and isotropic universe, the spatial derivatives of $R$ vanish, leading to a chemical potential for each lepton generation of the form

$$
\mu_{i}=\dot{R} \sum_{\alpha, \beta, j} \frac{\operatorname{Im}\left[\lambda_{\beta i}^{\dagger} \lambda_{i \alpha} \lambda_{\beta j}^{\dagger} \lambda_{j \alpha}\right]}{3 M_{\alpha} M_{\beta}} I_{[\alpha \beta]} .
$$

This changes the equilibrium distributions of leptons and antileptons and, after summing over lepton generations, we get a total lepton asymmetry ${ }^{3}$

$$
n_{L}=\frac{T^{2} g_{\ell}}{6} \dot{R} \sum_{\alpha, \beta} \frac{\operatorname{Im}\left[\left(\lambda^{\dagger} \lambda\right)_{\alpha \beta}^{2}\right]}{3 M_{\alpha} M_{\beta}} I_{\alpha \beta} .
$$

Previously, C and CP violating operators of this kind have been added by hand [1015], with the assumption that they may arise from a complete theory of gravity. However, without an obvious source of $\mathrm{C}$ and $\mathrm{CP}$ violation in the underlying theory, it remains unclear how these operators would actually arise in an effective theory of quantum gravity, and with what magnitude. Instead, we demonstrate here how they are generated in a simple and elegant fashion directly from loop effects in a BSM quantum field theory in curved spacetime. Furthermore, we have a readily accountable source of CP violation from the Yukawa couplings.

We will describe this effect in great detail in the remainder of this paper, but for now, we emphasise three crucial radiatively-induced effects which allow the realisation of our mechanism and the generation of the operator (1.5).

1. As in the traditional scenario, it is only at the loop level that one becomes sensitive to the complex phases of the Yukawa couplings, so that $\mathrm{C}$ and $\mathrm{CP}$ violating operators such as (1.5) are only produced above some minimum number of loops needed to expose the $\mathrm{CP}$ violation.

2. The operator (1.5) manifestly violates the strong equivalence principle (SEP), allowing a distinction between the gravitational effect on different particles. Minimal coupling of the Lagrangian ensures that at tree level, the SEP still holds. However, at the loop level, the insertion of curved space propagators in figure 2 means that virtual particles probe the details of the background, causing the leptons to become sensitive to curvature effects. As a result, even in an inertial frame, radiative effects force the particle to become sensitive to curvature, permitting the existence of SEP violating operators (1.5). The interpretation of this effect is that the screening cloud causes the lepton to acquire an effective size and experience tidal forces, realised by couplings of various curvature tensors to the lepton fields in the effective action, such as (1.5). Together with $\mathrm{C}$ and $\mathrm{CP}$ violation, this SEP-violating effect also distinguishes between the leptons and antileptons.

\footnotetext{
${ }^{3}$ See, for example, [9] appendix $\mathrm{C}$ for a compendium of useful formulae for number and energy densities for particles of different statistics in thermal equilibrium.
} 


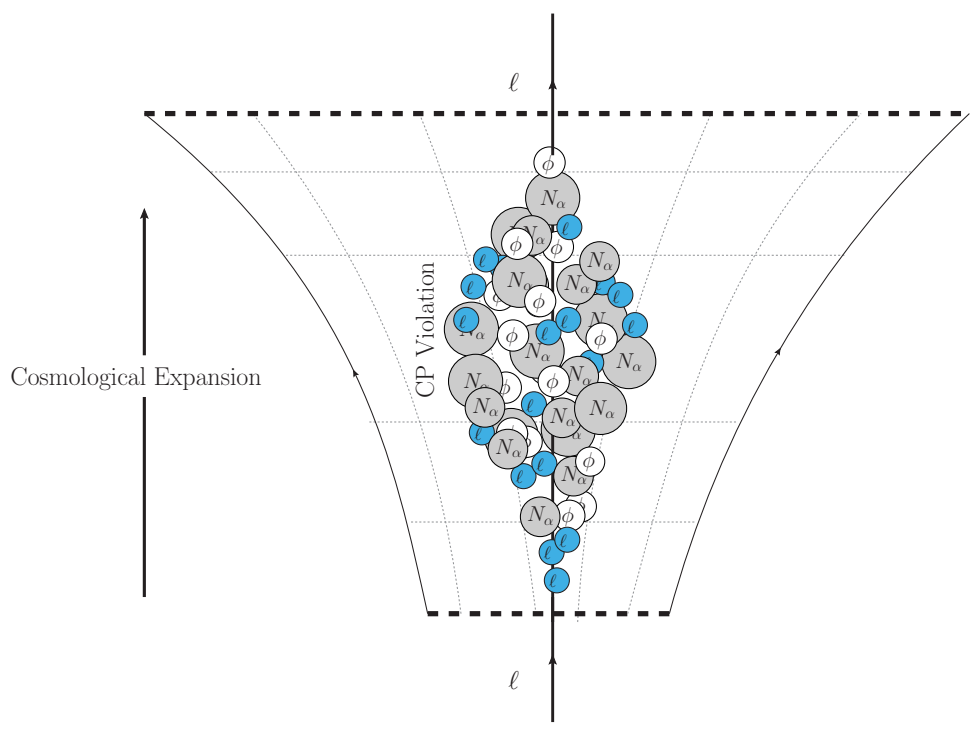

Figure 3. A schematic drawing illustrating how the lepton becomes sensitive both to CP violation and to the time-dependent nature of the background at the loop level.

3. In one form or another, time dependence of the dynamics is a necessary ingredient in any mechanism for producing a matter-antimatter asymmetry. In the sterile neutrino decay mechanism, this is realised according to the conventional Sakharov condition by a non-equilibrium process. In contrast, gravitational leptogenesis introduces time dependence through the coupling to an evolving background gravitational field [10]. The novel feature of our mechanism is that this sensitivity to the background arises dynamically, through explicitly quantum field theoretic effects occurring naturally at loop level. This elucidates why the operator (1.5) responsible for the matterantimatter asymmetry depends on the non-vanishing of the time derivative of the curvature. In this sense, the leptons inherit the time-dependent dynamics of the background. This is illustrated schematically in figure 3 .

Having highlighted some of the key similarities and differences between the traditional mechanism and our new approach, as embodied by the formulae (1.3) and (1.7) respectively, we spend the remainder of this paper examining in greater detail the origin of our effect, beginning with a discussion of propagation.

\section{Matter-antimatter propagation in flat and curved spacetime}

We begin with the following motivation, namely that the operator (1.5) causes a splitting in the spectra of matter and antimatter. Consider a quasi-plane wave solution for the lepton field $\ell(x)=u(x) e^{-i \Theta(x)}$, so that the momentum of the particle is given by $p_{\mu}=\partial_{\mu} \Theta$. As discussed in [16], when an operator of the form $b \partial_{\mu} R j^{\mu}$, coupling the derivative of the Ricci scalar to the lepton current, is added to the Dirac action, it gives different dispersion relations for particles and antiparticles. To see this, note that the modified equations of 
motion

$$
i \not D \ell+b \partial_{\mu} R \gamma^{\mu} \ell=0, \quad i \not D \ell^{c}-b \partial_{\mu} R \gamma^{\mu} \ell^{c}=0
$$

are solved in the context of the eikonal approximation [16] by

$$
\ell \sim u(x) e^{-i(\Theta-b R)}, \quad \ell^{c} \sim v(x) e^{-i(\Theta+b R)}
$$

leading to the dispersion relations

$$
\left(p_{\mu} \pm b \partial_{\mu} R\right)^{2}=0
$$

for the leptons and antileptons respectively. This causes a difference of energies between matter and antimatter - a picture which is consistent with the interpretation (1.6) of (1.5) as a chemical potential, which also corresponds to an energy-cost difference between particles and antiparticles.

We see then that the existence of this operator in an effective action necessarily implies that matter and antimatter propagate differently through the gravitational medium. This motivates the first step in the description of our mechanism, which involves a study of the propagation of matter and antimatter in flat and curved backgrounds.

\subsection{Propagation in translation invariant backgrounds}

In this section, we show, in two different ways, that matter and antimatter must propagate identically in translation invariant backgrounds. First, consider the transition amplitude for a particle to propagate between $x$ and $x^{\prime}$ :

$$
f_{s}\left(x^{\prime}, x\right)=\left\langle\psi\left(x^{\prime}\right), s \mid \psi(x), s\right\rangle
$$

Here, $\psi\left(x^{\prime}\right)$ and $\psi(x)$ denote a particle at $x$ and $x^{\prime}$, and $s$ labels helicity (spin) for massless (massive) particles. The corresponding amplitude for antiparticles is

$$
f_{s}^{c}\left(x^{\prime}, x\right)=\left\langle\psi^{c}\left(x^{\prime}\right), s \mid \psi^{c}(x), s\right\rangle,
$$

where the $C$ superscript denotes charge conjugate states. CPT symmetry is realised by an anti-unitary operator $\Theta$ in such a way that the inner product, represented by the bracket notation, is preserved under the action of $\Theta$ on each argument, together with an overall complex conjugation [17], i.e.,

$$
\left\langle\psi\left(x^{\prime}\right), s \mid \psi(x), s\right\rangle=\left\{\left(\left\langle\psi\left(x^{\prime}\right), s\right| \Theta\right)(\Theta|\psi(x), s\rangle)\right\}^{*} .
$$

Since $s$ is odd under CPT, we have $\Theta|\psi(x), s\rangle=\left|\psi^{c}(-x),-s\right\rangle$, and so, after complex conjugating, we get

$$
\left\langle\psi\left(x^{\prime}\right), s \mid \psi(x), s\right\rangle=\left\langle\psi^{c}(-x),-s \mid \psi^{c}\left(-x^{\prime}\right),-s\right\rangle \equiv f_{-s}^{c}\left(-x,-x^{\prime}\right),
$$

where in the rhs, we used the definition (2.5). Finally, we invoke translation symmetry which implies $f_{-s}^{c}\left(x^{\prime}, x\right)=f_{-s}^{c}\left(x^{\prime}-x\right)$, i.e., the transition amplitudes are functions of the 
relative position of the two points. This means that $f_{-s}^{c}\left(x^{\prime}, x\right)=f_{-s}^{c}\left(-x,-x^{\prime}\right)$ and hence, from (2.7), that

$$
f_{s}\left(x^{\prime}, x\right)=f_{-s}^{c}\left(x^{\prime}, x\right),
$$

establishing that the transition amplitude for a particle with spin/helicity $s$ to go from $x$ to $x^{\prime}$, is the same as the transition amplitude for an antiparticle with spin/helicity $-s$ to go from $x$ to $x^{\prime}$.

This is precisely the relevant statement for leptogenesis, since neutrinos (antineutrinos) have positive (negative) helicity, and shows that neutrinos and antineutrinos propagate identically in translation invariant backgrounds. Similarly, for massive particles, this would mean that spin up (down) particles propagate the same as spin down (up) antiparticles respectively, so that, averaging over spins, there is no difference in propagation. Notice the result (2.8) is non-perturbative and holds generically in any theory satisfying translation invariance.

We now demonstrate this result explicitly in the see-saw model at the correlator level by studying the lepton and antilepton propagators (including radiative corrections) given by

$$
S_{a b}\left(x^{\prime}, x^{\prime}\right)=\left\langle\ell_{a}\left(x^{\prime}\right) \bar{\ell}_{b}(x)\right\rangle, \quad S_{a b}^{c}\left(x^{\prime}, x\right)=\left\langle\ell_{a}^{c}\left(x^{\prime}\right) \overline{\ell_{b}^{c}}(x)\right\rangle,
$$

where the charge conjugate is given by $\ell=C(\bar{\ell})^{T}$ and $\overline{\ell^{c}}=-\ell^{T} C^{-1}$, and the matrix C satisfies $C\left(\gamma^{\mu}\right)^{T} C^{-1}=-\gamma^{\mu}$. As before, remembering that the Dirac bra-ket notion represents an inner product, the action of $\mathrm{CPT}$ on the propagator is

$$
\begin{aligned}
S_{a b}\left(x^{\prime}, x\right) & =\left\langle\ell_{a}\left(x^{\prime}\right) \bar{\ell}_{b}(x)\right\rangle \\
& =\left\langle\left(\Theta \ell_{a}\left(x^{\prime}\right) \Theta^{-1}\right)\left(\Theta \bar{\ell}_{b}(x) \Theta^{-1}\right)\right\rangle^{*},
\end{aligned}
$$

where

$$
\Theta \ell\left(x^{\prime}\right) \Theta^{-1}=\gamma^{0} \gamma_{5} C^{-1} \ell^{c}(-x) \quad \Theta \bar{\ell}\left(x^{\prime}\right) \Theta^{-1}=\overline{\ell^{c}}\left(-x^{\prime}\right) C \gamma_{5} \gamma^{0} .
$$

After performing the overall complex conjugation and, with a little algebra, we arrive at

$$
S\left(x^{\prime}, x\right)=\gamma_{5} C\left[S^{c}\left(-x,-x^{\prime}\right)\right]^{T} C^{-1} \gamma_{5} \text {. }
$$

As before, translation symmetry allows us to write $S^{c}\left(x^{\prime}, x\right)=S^{c}\left(x^{\prime}-x\right)$ and permits a momentum space representation

$$
S^{c}\left(x^{\prime}, x\right)=\int \frac{d^{n} p}{(2 \pi)^{n}} S^{c}(p)^{-i p \cdot\left(x^{\prime}-x\right)} .
$$

Furthermore, by Lorentz symmetry we must have

$$
S^{c}(p)=A\left(p^{2}\right) \not p+B\left(p^{2}\right),
$$

for some functions $A$ and $B$, which in general will depend also on $\lambda, M_{\alpha}$ and $M_{\beta}$ for the full propagator. Inserting (2.14) and (2.13) into (2.9), we find, after a little matrix manipulation, that

$$
S\left(x^{\prime}, x\right)=S^{c}\left(x^{\prime}, x\right) .
$$




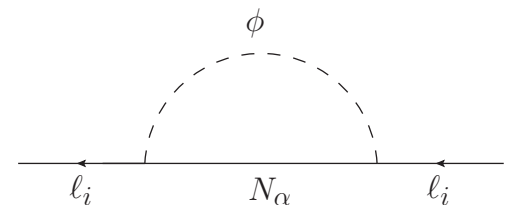

Figure 4. One-loop lepton self-energy.
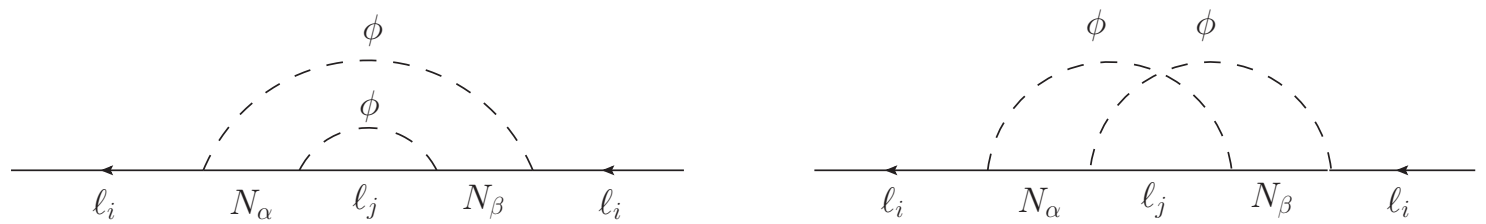

Figure 5. Two loop diagrams in curved space which give $\Sigma\left(x^{\prime}, x\right)-\Sigma^{c}\left(x^{\prime}, x\right) \neq 0$.

This reproduces the same result (2.8) at the level of correlators, showing that translational invariance forbids a difference in lepton and antilepton propagators. Notice that at the correlator level, spin seems not to enter the proof. This is because, although spin is exchanged under CPT, the field operator is a superposition over spin states, so that the flip in spin becomes absorbed into this sum.

As a consequence of the above results, there can be no asymmetric propagation of matter and antimatter in flat space. Conversely, when we relax the constraint of translation invariance, as happens in general gravitational backgrounds, we should expect to see a difference in the propagation of matter and antimatter.

\subsection{Propagation in curved backgrounds}

As discussed in section 1.1, loop effects lead to a violation of the SEP, which forces lepton propagators to become sensitive to a breaking of translational invariance by the background. Of course, to have a difference in the propagation of leptons and antileptons, it is necessary also to break $\mathrm{C}$ and $\mathrm{CP}$ violation, which is achieved via the complex phases in $\lambda_{i \alpha}$. We now demonstrate explicitly that when these three symmetries are broken, there is a difference in the propagation of matter and antimatter. We study this in terms of the self-energies $\Sigma\left(x^{\prime}, x\right)$ and $\Sigma^{c}\left(x^{\prime}, x\right)$ associated to the lepton and antilepton propagators of $(2.9)$.

At one loop (see figure 4), we immediately see $\Sigma\left(x^{\prime}, x\right)-\Sigma^{c}\left(x^{\prime}, x\right)=0$, with

$$
\Sigma_{i}\left(x^{\prime}, x\right)=\Sigma_{i}^{c}\left(x^{\prime}, x\right)=\sum_{\alpha} \lambda_{\alpha i}^{\dagger} \lambda_{i \alpha} G\left(x^{\prime}, x\right) S_{\alpha}\left(x^{\prime}, x\right)
$$

since the couplings occur only in the combination $\left(\lambda^{\dagger} \lambda\right)_{i j}$ for which the diagonal elements are manifestly real.

However, at two loops, there are two diagrams (figure 5) which give non-zero contributions to $\Sigma\left(x^{\prime}, x\right)-\Sigma^{c}\left(x^{\prime}, x\right)$. For instance, in the case of the charge-violating heavy neutrino 
propagators (see section 1.1), the diagram on the left gives

$$
\begin{aligned}
& \Sigma_{i}\left(x^{\prime}, x\right)-\Sigma_{i}^{c}\left(x^{\prime}, x\right) \\
& \quad=\sum_{\alpha, \beta, j} 2 i \operatorname{Im}\left[\lambda_{\beta i}^{\dagger} \lambda_{i \alpha} \lambda_{\beta j}^{\dagger} \lambda_{j \alpha}\right] G\left(x^{\prime}, x\right) \int d^{d} y \int d^{d} z G(y, z) S_{[\alpha}^{\times}\left(x^{\prime}, y\right) S_{j}(y, z) S_{\beta]}^{\times}(z, x),
\end{aligned}
$$

while the one on the right yields

$$
\begin{aligned}
& \Sigma_{i}\left(x^{\prime}, x\right)-\Sigma_{i}^{c}\left(x^{\prime}, x\right) \\
& \quad=\sum_{\alpha, \beta, j} 2 i \operatorname{Im}\left[\lambda_{\beta i}^{\dagger} \lambda_{i \alpha} \lambda_{\beta j}^{\dagger} \lambda_{j \alpha}\right] \int d^{d} y \int d^{d} z G\left(y, x^{\prime}\right) G(x, z) S_{[\alpha}^{\times}\left(x^{\prime}, y\right) S_{j}(y, z) S_{\beta]}^{\times}(z, x) .
\end{aligned}
$$

Notice that we have antisymmetrised over $\alpha$ and $\beta$ in the integral since $\operatorname{Im}\left[\lambda_{\beta i}^{\dagger} \lambda_{i \alpha} \lambda_{\beta j}^{\dagger} \lambda_{j \alpha}\right]$ is antisymmetric in $\alpha, \beta$.

For the other type of heavy neutrino propagator, only the first diagram exists (due to $\mathrm{SU}(2)_{L}$ charge considerations) and it has a different Yukawa index structure:

$$
\begin{aligned}
& \Sigma_{i}\left(x^{\prime}, x\right)-\Sigma_{i}^{c}\left(x^{\prime}, x\right) \\
& \quad=\sum_{\alpha, \beta, j} 2 i \operatorname{Im}\left[\lambda_{\beta i}^{\dagger} \lambda_{i \alpha} \lambda_{\alpha j}^{\dagger} \lambda_{j \beta}\right] G\left(x^{\prime}, x\right) \int d^{d} y \int d^{d} z G(y, z) S_{[\alpha}\left(x^{\prime}, y\right) S_{j}(y, z) S_{\beta]}(z, x) .
\end{aligned}
$$

As we see later, however, this does not contribute to the overall lepton number asymmetry.

It is now clear that eqs. (2.17), (2.18) and (2.19) are non-vanishing in curved spacetime. We see, therefore, that at two loops, leptons and antileptons propagate differently, due to a breaking of translation invariance by a general background, which is probed by curvedspace Green functions, $S_{j}(x, y), G(x, y)$ and $S_{\alpha}(x, y)$. In diagrammatic terms, this is how the time dependence necessary to evade the theorems in section 2.1 arises in our mechanism for leptogenesis. From a calculational point of view, the breaking of translation symmetry causes the two-loop self-energy to become sensitive to the ordering of the sterile neutrinos, $N_{\alpha}$ and $N_{\beta}$, within it, which are distinguishable by virtue of their non-degenerate masses and provides an antisymmetric part to the Feynman integral.

Given the arguments of section 2.1, we must also find that, if we restore translation invariance by going to Minkowski space, the differences (2.17)-(2.19) will vanish. Indeed, it is easy to check that by substituting flat space propagators, the difference in self-energies is zero. For instance, substituting flat space propagators into (2.18) gives (see (1.2))

$$
\begin{aligned}
\Sigma( & \left(p, \eta_{\mu \nu}\right)-\Sigma^{c}\left(p, \eta_{\mu \nu}\right) \\
= & \sum_{\alpha, \beta, j} 2 i \operatorname{Im}\left[\lambda_{\beta i}^{\dagger} \lambda_{i \alpha} \lambda_{\beta j}^{\dagger} \lambda_{j \alpha}\right] \\
& \times\left(\int \frac{d^{d} k}{(2 \pi)^{n}} \int \frac{d^{d} \ell}{(2 \pi)^{n}} \frac{1}{(k-p)^{2}-m_{\mathrm{H}}^{2}} \frac{1}{(\ell-p)^{2}-m_{\mathrm{H}}^{2}} \frac{M_{\alpha}}{k^{2}-M_{\alpha}^{2}} \frac{\not k+\ell-\not p}{(k+\ell-p)^{2}} \frac{M_{\beta}}{\ell^{2}-M_{\beta}^{2}}-(\alpha \leftrightarrow \beta)\right) \\
= & 0,
\end{aligned}
$$


where, after a trivial relabeling of integration variables, the expression is easily seen to be symmetric under interchange of $M_{\alpha}$ and $M_{\beta}$, and hence zero. A similar result holds for the other diagrams.

We now show how this difference in propagation manifests itself in the form of curvature-dependent, $\mathrm{C}$ and $\mathrm{CP}$ violating operators in the effective action generated by the diagrams in figure 5 .

\section{Effective field theory — integrating out the sterile neutrinos}

One of the most direct ways to study the propagation of particles in gravitational backgrounds, and the effects of curvature on their dynamics, is to use effective field theory $[16,18,19]$. As discussed in the previous sections, the screening cloud surrounding an interacting particle gives it an effective size, causing it to experience tidal forces. When one integrates out the heavy sterile neutrinos, this phenomenon generates operators in the effective action that couple particle fields to various curvature tensors, suppressed by a seesaw scale cutoff. The most general such action [16], constructed from a complete basis of hermitian operators, is given to linear order in curvature and leading order in derivatives by

$$
\begin{aligned}
\mathcal{L}_{\mathrm{eff}}=\sqrt{-g}[\bar{\ell} i \not D \ell & +i a \bar{\ell}\left(2 R_{\mu \nu} \gamma^{\mu} D^{\nu}+\frac{1}{2} \partial_{\mu} R \gamma^{\mu}\right) \ell+b \partial_{\mu} R \bar{\ell} \gamma^{\mu} \ell \\
& \left.+i c \bar{\ell}\left(2 R \not D+\partial_{\mu} R \gamma^{\mu}\right) \ell+i d \bar{\ell}\left(2 D^{2} \not D+\frac{1}{4} \partial_{\mu} R \gamma^{\mu}\right) \ell\right]
\end{aligned}
$$

where $a, b, c, d$ are real, effective couplings, with mass dimension minus two, which will depend on $\lambda_{i \alpha}$ and the masses $M_{\alpha}$ and $M_{\beta}$ in the loops. There is one term in this effective action which is of great importance for leptogenesis, and is the only $\mathrm{C}$ and $\mathrm{CP}$ violating operator in (3.1), viz.

$$
\mathcal{L}_{\mathrm{CPV}}=b \partial_{\mu} R \bar{\ell} \gamma^{\mu} \ell
$$

A careful discussion of the action of $\mathrm{C}, \mathrm{P}$ and $\mathrm{T}$ on this, and remaining operators appearing in $\mathcal{L}_{\text {eff }}$ was given in [16].

The presence of a $\mathrm{C}$ and $\mathrm{CP}$ violating operator ensures that the second Sakharov condition holds, namely that the complete theory contains $\mathrm{C}$ and $\mathrm{CP}$ violation, which is provided here by the complex couplings $\lambda_{i \alpha}$. In our model, this means that the operator will be generated at the two-loop level, by precisely the same diagrams which lead to asymmetric propagation of matter and antimatter, and will depend on the complex phases contained in the Yukawa couplings $\lambda_{i \alpha}$. In the next section, we show how integrating out the heavy neutrinos from these diagrams gives an expression for the effective coupling $b$, whose calculation we shall now describe in detail.

At this point, we should comment further on the range of validity of the effective Lagrangian (3.1) in the context of leptogenesis. It is written to first order in the curvatures, so is valid for small values of the parameter $\mathfrak{R} / M^{2}$, where $\mathfrak{R}$ denotes a typical curvature component while $M$ is the heavy scale, provided here by the sterile neutrino masses. It is also a low-energy Lagrangian, keeping only terms of leading order in derivatives. As shown 


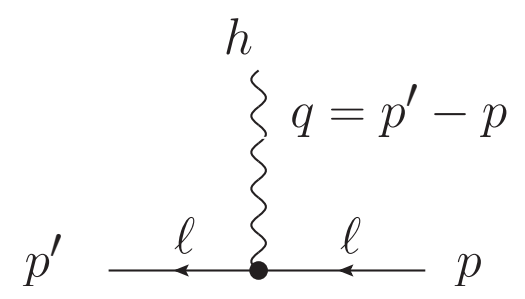

Figure 6. The effective $h$ vertex generated by $\mathcal{L}_{\mathrm{CPV}}$ where $q=p^{\prime}-p$ is the momentum transfer between the ingoing and outgoing lepton.

in the series of papers [20-22] which discuss the realisation of causality and the energy dependence of theories of this type, the relevant parameter here is $E \sqrt{\mathfrak{R}} / M^{2}$, where $E$ denotes the lepton energy scale. Both these parameters are required to be small for the validity of the effective Lagrangian. We return to this point in section 5, where we apply the effective Lagrangian and chemical potential to the hot, early Universe where both $E$ and $\mathfrak{R}$ are related to the temperature.

\subsection{Matching}

The calculation of the effective couplings in (3.1) is performed in the usual way, by matching the fundamental UV-complete theory to the effective action at low energy. In particular, the calculation can be reduced to the problem of evaluating a certain class of two-loop Feynman diagrams, which we now describe. The first step is to notice that the effective couplings are independent of the choice of background, so that a judicious choice of metric greatly simplifies the computation. We shall pick a conformally flat metric

$$
g_{\mu \nu}=\Omega^{2} \eta_{\mu \nu}=(1+h) \eta_{\mu \nu}
$$

which is sufficient to distinguish the various components of the effective Lagrangian (3.1). In particular, since the Ricci tensor is given by

$$
R_{\mu \nu}=-\partial_{\mu} \partial_{\nu} h-\frac{1}{2} \eta_{\mu \nu} \partial^{2} h+\mathcal{O}\left(h^{2}\right)
$$

we see that the effective couplings can be determined by working to linear order in $h$. For instance, since $R=-3 \partial^{2} \Omega^{2}$, the contribution to the effective vertex from the operator $\mathcal{L}_{\mathrm{CPV}}=b \partial_{\mu} R \bar{\ell} \gamma^{\mu} \ell$, is given by

$$
\mathcal{L}_{\mathrm{CPV}}=-3 b\left(\partial_{\mu} \partial^{2} h\right) \bar{\ell} \gamma^{\mu} \ell+O\left(h^{2}\right) .
$$

We can then use the Minkowski background to define a momentum space, over which $h$ is treated as a classical background field. This gives a contribution from this operator of the form

$$
A(q)=3 i b\left(q^{2} q\right) h(q)
$$

which corresponds to the diagram in figure 6 . 
Similarly we can expand the Lagrangian of the UV theory

$$
\mathcal{L}=\mathcal{L}_{E W}+\sqrt{-g}\left[\bar{N} i \not D N+\lambda_{i \alpha} \bar{\ell}_{i} \phi N_{\alpha}+\frac{1}{2} \overline{\left(N^{c}\right)} M N+\text { h.c. }\right]
$$

to linear order in $h$. The computation is also simplified if we work with conformally rescaled fields,

$$
N \rightarrow \Omega^{-(n-1) / 2} N, \quad \ell \rightarrow \Omega^{-(n-1) / 2} \ell, \quad \phi \rightarrow \Omega^{-(n-2) / 2} \phi .
$$

After conformal rescaling of the Lagrangian (3.7) and inserting the metric (3.3), gravity enters only via the terms which violate conformal invariance, so that the $\Omega$-dependent terms in the Lagrangian can be written as

$$
\begin{aligned}
\mathcal{L}_{\Omega} & =\frac{1}{2} \Omega \overline{N^{c}} M N+\Omega^{2}\left(m_{\mathrm{H}}^{2}-6\left(\zeta-\frac{1}{6}\right) \Omega^{-3} \partial^{2} \Omega\right) \phi^{\dagger} \phi+\Omega^{-(n-4) / 2} \lambda_{i \alpha} \bar{\ell}_{i} \phi N \\
& =\mathcal{O} h+O\left(h^{2}\right),
\end{aligned}
$$

where the Higgs Lagrangian includes the Ricci coupling $\zeta R \phi^{\dagger} \phi$. Expanding to linear order in $h$, we have

$$
\mathcal{O} h=\frac{1}{4} h M \bar{N} N^{c}+\text { h.c. }+\left(m_{\mathrm{H}}^{2} h-3\left(\zeta-\frac{1}{6}\right) \partial^{2} h\right) \phi^{\dagger} \phi-\frac{(n-4)}{4} h \lambda_{i \alpha} \bar{\ell}_{i} \phi N .
$$

In this way, gravity manifests itself in the form of a classical background field $h$, so that the two-loop diagrams can be expanded to linear order in $h$ by appropriate insertion of $h$ according to the operators in (3.10). This reduces the problem to the evaluation of flat space 3-point Feynman diagrams, with two external fermion legs and a classical field $h$.

For the case of $h$ couplings to the heavy neutrinos via their mass term, the corresponding diagrams are shown in figure 7 . Suppose that the $h$ insertion is made into the $N_{\alpha}$ propagator. Recalling that only the diagrams with both propagators of the chargeviolating type contribute to leptogenesis, the $N_{\beta}$ propagator must be of the type $S_{\beta}^{\times}$ in (1.2). Then, given the two terms in (3.10) for the coupling of $h$ to the sterile neutrinos, viz. $\frac{1}{4} h M\left(\bar{N} N^{c}+\overline{N^{c}} N\right)$, we see that with this condition the $N_{\alpha}$ line receives contributions from both $S_{\alpha} S_{\alpha}^{c}$ and $S_{\alpha}^{\times} S_{\alpha}^{\times}$. Finally, we use $S_{\alpha}^{c}=S_{\alpha}$ in flat space. This establishes the form of the diagrams to be calculated in the following section.

The effective couplings can be computed by matching the transition matrix elements $\left\langle\ell\left(p^{\prime}\right)|\mathcal{O} h| \ell(p)\right\rangle$ for small external momenta to the effective amplitudes such as (3.6) (see in particular $[16,19]$, as well as $[18,23-25]$, for more details). The general form of this object is

$$
\left\langle\ell_{i}\left(p^{\prime}\right)|\mathcal{O} h| \ell_{i}(p)\right\rangle=\not p\left[\alpha_{1} p^{2}+\alpha_{2}(p \cdot q)+\alpha_{3} q^{2}\right]+\not d\left[\beta_{1} p^{2}+\beta_{2}(p \cdot q)+\beta_{3} q^{2}\right]
$$

where $q=p^{\prime}-p$ is the momentum transfer, and $\alpha_{i}$ and $\beta_{i}$ are in general complex coefficients, which depend on the Yukawa couplings and the masses in the loop. From (3.6), we see that the coefficient $b$ can be read off as $b=1 / 3 \operatorname{Im}\left(\beta_{3}\right)$, which in turn only depends on the value of the momentum transfer $q$. Hence, for the purposes of calculating the operator (3.2), we 

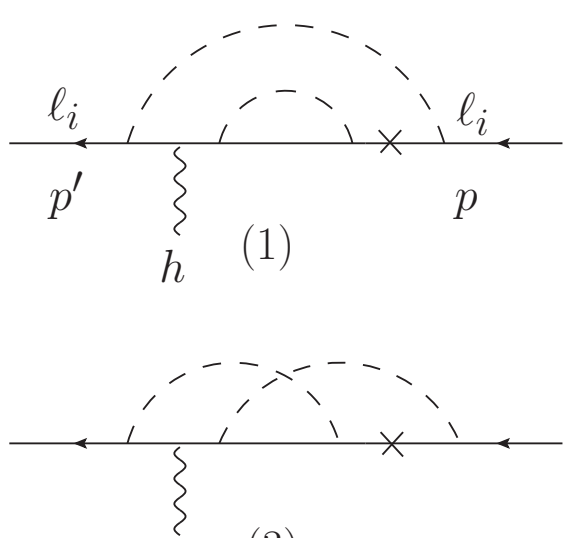

(3)
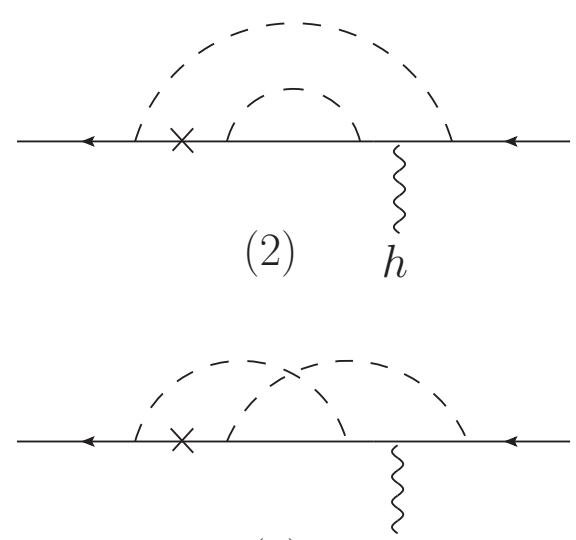

(4)

Figure 7. Contributions to $\left\langle\ell\left(p^{\prime}\right)|\mathcal{O} h| \ell(p)\right\rangle$ from the heavy neutrino mass term $\frac{1}{2} h \bar{N} M N^{c}$. The crosses denote the $S_{\beta}^{\times}$sterile neutrino propagator, while on the lines with $h$ insertions there are contributions $S_{\alpha} S_{\alpha}$ and $S_{\alpha}^{\times} S_{\alpha}^{\times}$corresponding to each propagator type.

can set $p=0$ in the remainder of our calculations, so that the amplitudes are functions of a single momentum $q$, with $p^{\prime}=q$. The transition amplitude is thus given by

$$
\begin{aligned}
\left\langle\ell_{i}(q)|\mathcal{O} h| \ell_{i}(0)\right\rangle & =\sum_{\alpha, \beta, j} \lambda_{\beta i}^{\dagger} \lambda_{i \alpha} \lambda_{\beta j}^{\dagger} \lambda_{j \alpha} f\left(q^{\mu}, M_{\alpha}, M_{\beta}\right) \\
& =i q^{2} \not h h(q) \sum_{\alpha, \beta, j} \frac{\operatorname{Im}\left[\lambda_{\beta i}^{\dagger} \lambda_{i \alpha} \lambda_{\beta j}^{\dagger} \lambda_{j \alpha}\right]}{M_{\alpha} M_{\beta}} I_{[\alpha \beta]}+\cdots,
\end{aligned}
$$

where $+\cdots$ represents terms which do not contribute to (3.6). The factor $I_{\alpha \beta}=I\left(M_{\alpha}, M_{\beta}\right)$ depends on the masses of the sterile neutrinos. It can be determined by performing a momentum expansion of $f\left(q, M_{\alpha}, M_{\beta}\right)$, in the limit $-q^{2} \ll M_{\alpha}, M_{\beta}$, where $f$ is directly determined from the evaluation of the three-point Feynman diagrams in figure 7 . In this sense, the see-saw scale becomes the UV cut-off for our effective theory. Matching the effective amplitude (3.6) to (3.12) we find that

$$
\mathcal{L}_{\mathrm{CPV}}=\partial_{\mu} R \bar{\ell}_{i} \gamma^{\mu} \ell_{i} \sum_{\alpha, \beta, j} \frac{\operatorname{Im}\left[\lambda_{\beta i}^{\dagger} \lambda_{i \alpha} \lambda_{\beta j}^{\dagger} \lambda_{j \alpha}\right]}{3 M_{\alpha} M_{\beta}} I_{[\alpha \beta]} .
$$

In this way, we have shown how this operator arises simply from curved space QFT considerations, without the need to postulate new gravitational interactions arising from some unspecified, more fundamental theory.

We shall describe the relevant calculations for $I_{\alpha \beta}$ shortly, but our key finding, which we state now for continuity, is that the size of the loop effects contributing to leptogenesis may be enhanced by increasing the hierarchy between $M_{\alpha}$ and $M_{\beta}$. Specifically, we find that keeping $M_{\alpha}$ fixed, the propagator correction diagrams (1) and (2) are suppressed for a large ratio $\xi=M_{\beta} / M_{\alpha}$, whereas the vertex correction diagrams grow linearly with $\xi$. For 

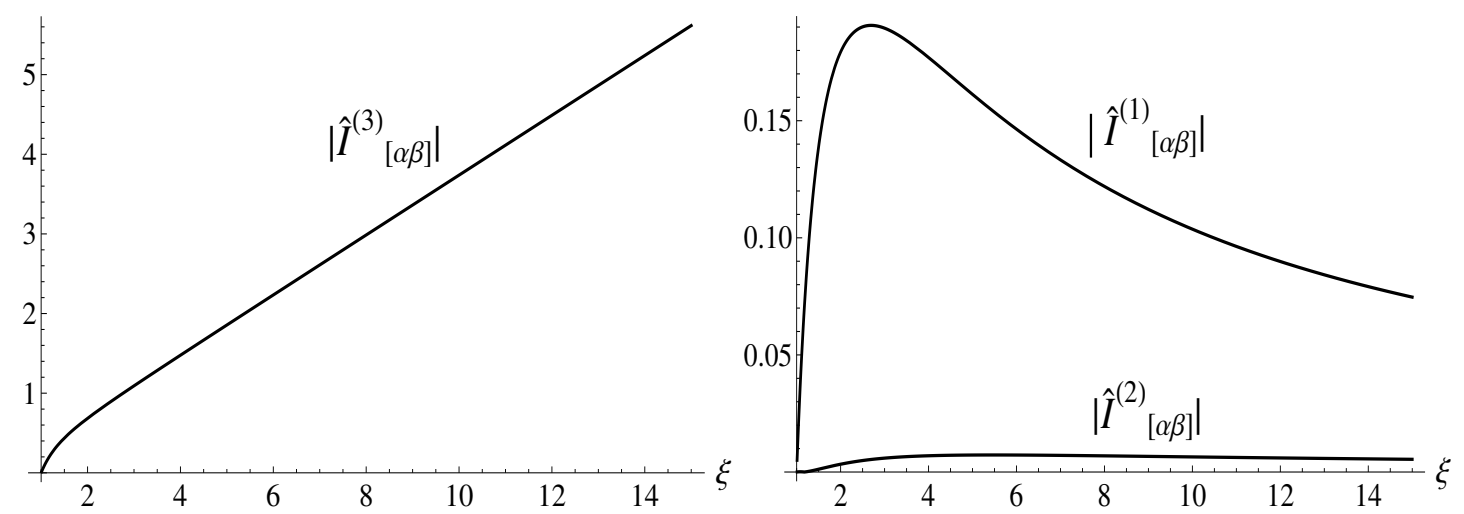

Figure 8. The hierarchy enhancement of loop effects, with $M_{\alpha}$ fixed. The curves show $\hat{I}_{[\alpha, \beta]}^{(n)}=$ $(4 \pi)^{4}\left|I_{[\alpha, \beta]}^{(n)}\right| / \xi$ plotted as a function of the heavy sterile neutrino mass ratio $\xi=M_{\beta} / M_{\alpha}$.

instance, we find that the loop contribution from the vertex correction diagram (3) gives

$$
\frac{I_{[\alpha \beta]}^{(3)}}{M_{\alpha} M_{\beta}} \simeq-\frac{3}{8} \frac{1}{(4 \pi)^{4}} \frac{1}{M_{\alpha}^{2}} \xi \quad \xi \equiv \frac{M_{\beta}}{M_{\alpha}} \gg 1,
$$

while for the propagator correction diagrams (1) and (2) we have

$$
\frac{I_{[\alpha \beta]}^{(1)}}{M_{\alpha} M_{\beta}} \simeq-\frac{5}{16} \frac{1}{(4 \pi)^{4}} \frac{1}{M_{\alpha}^{2}} \frac{1}{\xi}, \quad \frac{I_{[\alpha \beta]}^{(2)}}{M_{\alpha} M_{\beta}} \simeq-\frac{1}{24} \frac{1}{(4 \pi)^{4}} \frac{1}{M_{\alpha}^{2}} \frac{\ln (\xi)}{\xi},
$$

in the same limit. This behaviour is plotted in figure 8 .

The necessary 2-loop calculations, even in the low-momentum limit, are not simple, and considerable care must be taken in particular to deal with the various massless thresholds which arise. Note that it is only the terms of $O\left(q^{2} q\right)$ which contribute to the local effective Lagrangian (3.5). We also encounter non-analytic terms involving $\ln \left(-q^{2}\right)$, which are to be interpreted as non-local contributions to the effective action. While such terms are of considerable importance in their own right and encode important information about the long-range interactions in the theory $[24,25]$, they do not affect the leptogenesis mechanism of interest to us here. Full details of these calculations are presented in the next section. The reader interested primarily in the implications for cosmology may at this point jump ahead directly to section 5 , where the consequences for leptogenesis are discussed.

\section{Feynman diagram calculations}

We now describe the calculation of the two-loop self-energy diagrams diagrams shown in figure 7. Diagrams (1)-(3) can be evaluated by first evaluating the one-loop propagator or vertex sub-diagrams, which in these cases are relatively simple, then inserting into the full self-energy diagrams. However, the sub-diagram for (4) is of a non-trivial triangle type [26] and here the whole diagram must be dealt with in a different way. 


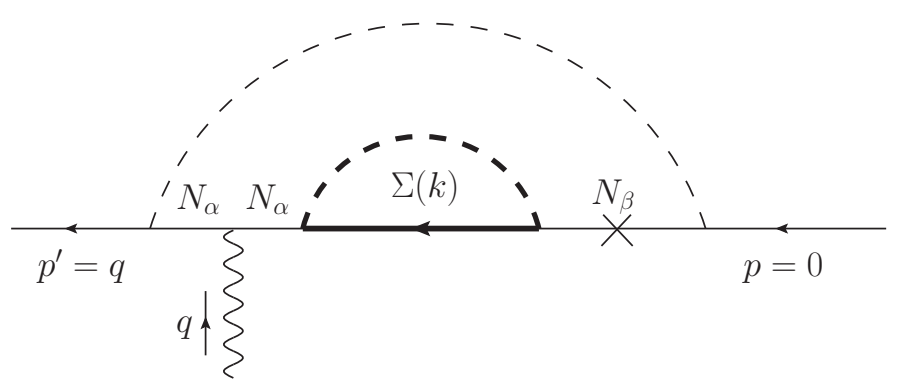

Figure 9. The propagator correction diagram with no branch cuts. The self-energy sub-diagram $\Sigma(k)$ is shown in bold and we sum over the two kinds of sterile neutrino propagators which can be inserted at the $h$ vertex.

\subsection{Sterile neutrino couplings}

First, we describe the diagrams where the gravitational field $h$ couples to the heavy sterile neutrino propagators.

Diagram (1). This diagram gives

$$
f^{(1)}\left(q^{\mu}, M_{\alpha}, M_{\beta}\right)=\frac{M_{\alpha}}{2} \int \frac{d^{d} k}{(2 \pi)^{d}} \int \frac{d^{d} \ell}{(2 \pi)^{d}} G(k)\left[S_{\alpha}(k+q) S_{\alpha}(k)+S_{\alpha}^{\times}(k+q) S_{\alpha}^{\times}(k)\right] \Sigma(k) S_{\beta}^{\times}(k),
$$

where $\Sigma(k)$ is the massless sub-diagram shown in bold in figure 9 :

$$
\Sigma(k)=\int \frac{d^{d} \ell}{(2 \pi)^{d}} G(k-\ell) S(\ell) .
$$

It is easily evaluated to give

$$
\Sigma(k)=\frac{i \not k}{(4 \pi)^{2}}\left[\frac{1}{2} \ln \left(-\frac{k^{2}}{\mu^{2}}\right)-1\right],
$$

where $\mu$ is an RG scale, and we used $\overline{M S}$ when removing the pole. One can then re-insert this into the main diagram to find $f^{(1)}\left(q, M_{\alpha}, M_{\beta}\right)$, which can be written as a momentum expansion in $q$. The details of this expansion and the techniques necessary to evaluate the full diagram are described in the appendix. One can then read off the contribution to $I_{\alpha \beta}$ and its dependence on the masses $M_{\alpha}$ and $M_{\beta}$. For this diagram, we find a contribution to $I_{\alpha \beta}$ given by

$$
\begin{aligned}
I_{\alpha \beta}^{(1)}=\frac{1}{24(4 \pi)^{4}(x-1)^{5}}[24 & \ln (x)\left(\left(x^{2}+x-1\right) \ln \left(\frac{\mu^{2}}{M_{\alpha}^{2}}\right)+x\right) \\
+ & 2(x-1)(x(x((x-5) x-19)+7)+4) \ln \left(\frac{\mu^{2}}{M_{\alpha}^{2}}\right) \\
& \left.-12\left(x^{2}+x-1\right) x \ln ^{2} x-3 x^{5}+28 x^{4}-54 x^{3}+41 x-12\right]
\end{aligned}
$$




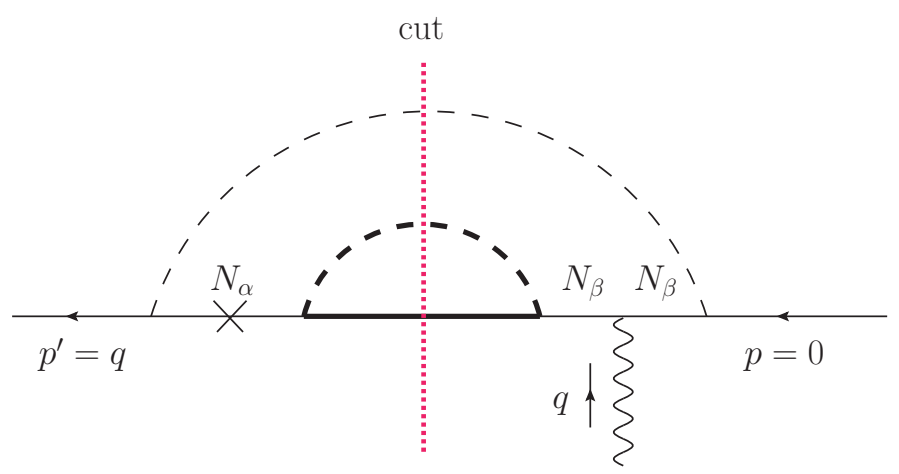

Figure 10. The propagator correction diagram with a zero-mass threshold corresponding to the cut (shown in red) through the massless Higgs and lepton lines, across which the momentum $q$ flows.

where

$$
x=\xi^{2}=\frac{M_{\beta}^{2}}{M_{\alpha}^{2}} .
$$

The large $x$ behaviour for the antisymmetric part, choosing the RG scale $\mu=M_{\alpha}$, is therefore given by

$$
I_{[\alpha, \beta]}^{(1)} \simeq-\frac{5}{16} \frac{1}{(4 \pi)^{4}}+O\left(\frac{\ln ^{2} x}{x}\right), \quad x \gg 1
$$

as quoted in (3.15).

Diagram (2). This diagram contains a zero-mass threshold [27-29] as shown by the cut in figure 10. As a result, the diagram has a discontinuity at $q^{2}=0$ and a branch cut represented by the appearance of $\ln \left(\frac{-q^{2}}{M_{\alpha}^{2}}\right)$ in the expansion for $f^{(2)}\left(q, M_{\alpha}, M_{\beta}\right)$. We find

$$
f^{(2)}\left(q, M_{\alpha}, M_{\beta}\right)=\frac{\not d}{M_{\alpha} M_{\beta}}\left[I_{\alpha \beta}^{(2)}-\frac{1}{24} \ln \left(-\frac{q^{2}}{M_{\alpha}^{2}}\right)\right] q^{2}+\cdots
$$

with $I_{\alpha \beta}^{(2)}$ given by

$$
\begin{aligned}
I_{\alpha \beta}^{(2)}=\frac{1}{144(4 \pi)^{4}(x-1)^{5}}[6 \ln & \left(\frac{\mu^{2}}{M_{\alpha}^{2}}\right)\left(2 x^{5}-11 x^{4}+28 x^{3}-20 x^{2}-12 x^{2} \ln (x)+2 x-1\right) \\
+ & 16 x^{5}-119 x^{4}+88 x^{3}-34 x^{2}+36 x^{2} \ln ^{2}(x) \\
& \left.-6\left(x^{4}-6 x^{3}-6 x^{2}-10 x-3\right) x \ln (x)+56 x-7\right] .
\end{aligned}
$$

In the large hierarchy limit, the antisymmetric part in this case is

$$
I_{[\alpha, \beta]}^{(2)} \simeq-\frac{1}{48} \frac{\ln x}{(4 \pi)^{4}}+O(1), \quad x \gg 1 .
$$




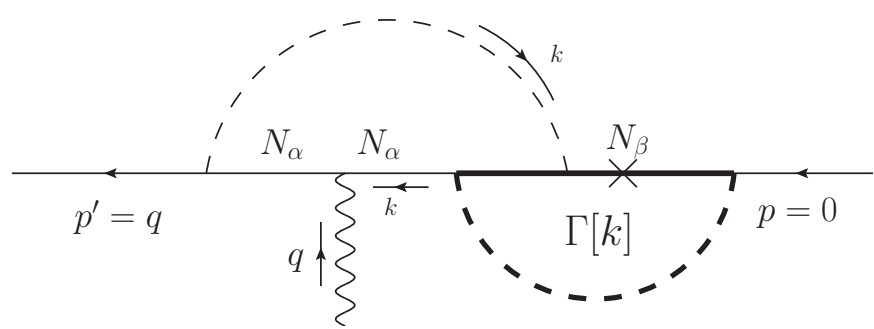

Figure 11. The vetex-correction diagram with no branch cuts. The vertex correction sub-diagram $\Gamma(k)$, is shown in bold, with a single momentum $k$ flowing in and back out.

Diagram (3). As before, the relevant subdiagram is shown in bold (figure 11).

Although this sub-diagram is of the triangle type [26] which, in general, gives a lengthy answer, since there is only one momentum flowing into it, and since it contains only one mass, the expression is relatively simple:

$$
\Gamma(k)=\frac{i \hbar k}{(4 \pi)^{2} M_{\beta}}\left\{\left[\frac{M_{\beta}^{4}}{k^{4}}-\frac{M_{\beta}^{2}}{k^{2}}\right] \ln \left(\frac{M_{\beta}^{2}-k^{2}}{M_{\beta}^{2}}\right)-\frac{M_{\beta}^{2}}{k^{2}}-\frac{1}{2} \ln \left(\frac{k^{2}-M_{\beta}^{2}}{k^{2}}\right)\right\} .
$$

Substituting this into the main diagram, one can evaluate the remaining $k$ integral following the procedure described in the appendix. There are no branch cuts in this diagram, and hence no $\ln \left(-q^{2}\right)$ terms. The final contribution is

$$
\begin{aligned}
I_{\alpha \beta}^{(3)}=\frac{1}{48(4 \pi)^{4}(x-1)^{4}}[- & 24(x-1)^{4}(2 x+1) x \operatorname{Li}_{2}\left(\frac{1}{x}\right)+30 x^{5}-67 x^{4}-16 x^{3}+153 x^{2} \\
+ & 2 x\left(24 x^{4}-71 x^{3}+58 x^{2}+4 x+12(2 x+1)(x-1)^{4} \ln (x-1)-24\right) \ln (x) \\
& \left.-122 x-24(x-1)^{4}(2 x+1) x \ln ^{2}(x)+22\right] .
\end{aligned}
$$

We are now in a position to derive the result (3.14). After antisymmetrisation, we find that the asymptotic behaviour of this diagram is given by

$$
I_{[\alpha \beta]}^{(3)} \simeq-\frac{3}{8} \frac{x}{(4 \pi)^{4}}+O(\ln x), \quad x \gg 1 .
$$

Diagram (4). The amplitude for this process is given by

$$
f^{(4)}\left(q, M_{\alpha}, M_{\beta}\right)=\frac{M_{\beta}}{2} \int \frac{d^{d} k}{(2 \pi)^{d}} \int \frac{d^{d} \ell}{(2 \pi)^{d}} \frac{M_{\alpha}(\not k+\ell)\left[M_{\beta}^{2}+\not k(k k-\not)\right]}{k^{2}\left[k^{2}-M_{\beta}^{2}\right] \ell^{2}\left[(k+\ell)^{2}\right]\left[(k-q)^{2}-M_{\beta}^{2}\right]\left[(\ell+q)^{2}-M_{\alpha}^{2}\right]},
$$

and corresponds to the diagram in figure 12 .

For this amplitude, the sub-diagram (shown in bold) is also of the triangle type. However in this instance it depends on two momenta, rather than just one. This vastly complicates the form of the sub-diagram, which is worthy of a separate analysis [26] in its own right. Furthermore, the overall diagram contains a branch cut, shown in red. Not only 


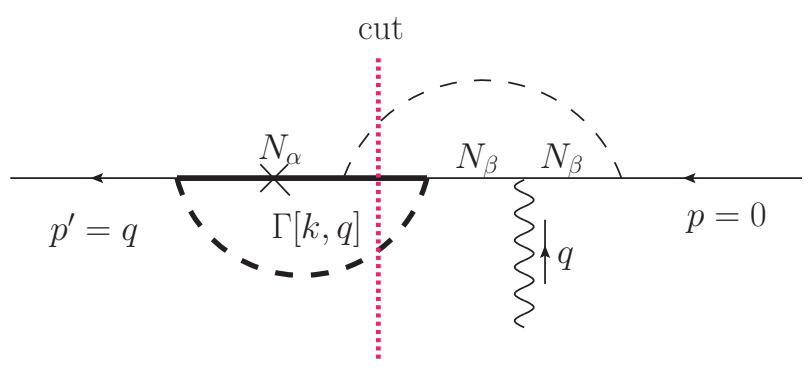

Figure 12. The vertex-correction diagram with a branch cut through Higgs and lepton lines.

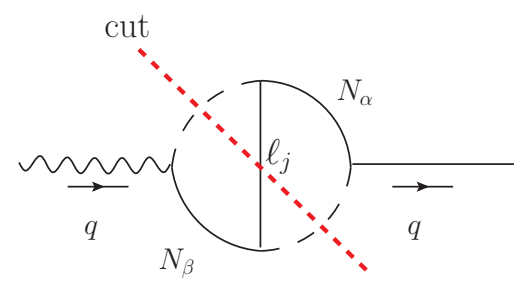

$f_{1}$

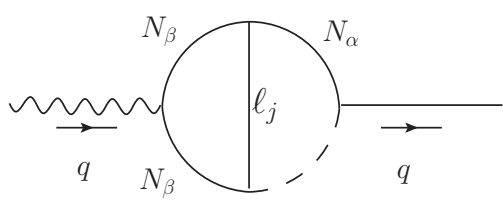

$f_{2}$

Figure 13. Self-energy diagrams resulting from partial fractioning.

that, but the complicated sub-diagram has to be substituted into the remaining momentum integral. Since we only require a momentum expansion, we shall proceed in a different way from the previous diagrams. First, we notice that we can use partial fractions to write

$$
\frac{1}{k^{2}} \cdot \frac{1}{k^{2}-M_{\beta}^{2}}=-\frac{1}{M_{\beta}^{2}}\left(\frac{1}{k^{2}}-\frac{1}{k^{2}-M_{\beta}^{2}}\right)
$$

which allows the diagram to be written as the difference of two self-energies of the types shown in figure 13. This allows us to write

$$
f^{(4)}\left(q, M_{\alpha}, M_{\beta}\right)=-\frac{1}{M_{\beta}^{2}}\left[f_{1}\left(q, M_{\alpha}, M_{\beta}\right)-f_{2}\left(q, M_{\alpha}, M_{\beta}\right)\right] .
$$

This class of diagrams has been extensively studied in the literature [27-31]. The first step is to simplify the gamma matrix expression in the numerator and write as far as possible as a sum of terms appearing in the propagator denominators. This allows the full diagram to be written as a sum of scalar diagrams with a smaller number of propagators, as shown below. Some of these are UV divergent, but the poles are all spurious, in the sense that their sum must be finite, as the overall diagram (4) contains no divergences. For instance, in the case of $f_{1}$, we find the diagram decomposes as

$$
\begin{aligned}
f_{1}=\frac{q}{2}\left[\frac{1}{2 q^{2}}\right. & \frac{M_{\alpha}}{M_{\beta}}(J(0,1,1,1,0)-J(0,1,1,0,1)-J(0,0,1,1,1)+J(-1,1,1,1,1)) \\
& +\frac{M_{\alpha}}{M_{\beta}}\left(\frac{M_{\beta}^{2}+M_{\alpha}^{2}}{2 q^{2}}-\frac{1}{2}\right) J(0,1,1,1,1)+\frac{M_{\alpha}}{2 M_{\beta}} J(1,0,1,1,1)-\frac{M_{\alpha}}{2 M_{\beta}} J(1,1,0,1,1)
\end{aligned}
$$




$$
\begin{aligned}
& -\frac{M_{\alpha} M_{\beta}}{q^{2}}[J(1,1,1,0,1)+J(1,0,1,1,1)]+\frac{M_{\alpha} M_{\beta}}{q^{2}}[J(1,1,1,1,0)+J(0,1,1,1,1)] \\
& \left.+\left(\frac{\left(M_{\alpha}^{2}+M_{\beta}^{2}\right)}{q^{2}}+\frac{1}{2}\right) M_{\alpha} M_{\beta} J(1,1,1,1,1)\right]
\end{aligned}
$$

where

$$
\begin{aligned}
& J\left(\nu_{1}, \nu_{2}, \nu_{3}, \nu_{4}, \nu_{5}\right) \\
& \quad=\int \frac{d^{d} k_{1}}{(2 \pi)^{d}} \int \frac{d^{d} k_{2}}{(2 \pi)^{d}} \frac{1}{\left[k_{1}^{2}-M_{\beta}^{2}\right]^{\nu_{1}}\left[k_{2}^{2}\right]^{\nu_{2}}\left[\left(k_{1}+k_{2}\right)^{2}\right]^{\nu_{3}}\left[\left(k_{1}-q\right)^{2}\right]^{\nu_{4}}\left[\left(k_{2}+q\right)^{2}-M_{\alpha}^{2}\right]^{\nu_{5}}} .
\end{aligned}
$$

The corresponding diagrams are given in figure 14. Not all these diagrams give a $\not q q^{2}$ contribution. Firstly, we note that diagram 2 is zero, since it contains a massless bubble, which vanishes in dimensional regularisation. Futhermore, diagram 3 has no $q$ dependence, and so does not contribute to $q^{2} \not$. We also see that last 2 lines of (4.16) give contributions which are symmetric under interchange of $\alpha$ and $\beta$, and so do not contribute to $I_{[\alpha \beta]}$. Hence there are only 5 diagrams which contribute: 1, 4, 5, 6 and 7. Diagrams 1 and 5 were computed by Scharf and Tausk [30] and yield ${ }^{4}$

$$
\begin{aligned}
J(0,1,1,1,0)= & -\frac{1}{(4 \pi)^{4}}\left[\frac{1}{4 \delta}-\frac{13}{8}+\frac{1}{2}\left\{\gamma+\ln \left(\frac{-q^{2}}{4 \pi \mu^{2}}\right)\right\}\right] \\
J(0,1,1,1,1)= & -\frac{1}{(4 \pi)^{4}}\left[\frac{1}{2 \delta^{2}}+\frac{1}{2 \delta}\left\{5-2 L_{\alpha}-2\left(\frac{1+Q}{Q}\right) \ln (1+Q)\right\}\right. \\
& +\frac{19}{2}+\frac{3}{2} \zeta(2)+L_{\alpha}^{2}-L_{\alpha}\left(5-2\left(\frac{1+Q}{Q}\right) \ln (1+Q)\right) \\
& -\ln (Q)+\left(\frac{1+Q}{Q}\right)\left\{2 \operatorname{Li}_{2}(-Q)+\ln ^{2}(1+Q)\right. \\
& +\ln (Q) \ln (1+Q)-4 \ln (1+Q)\}]
\end{aligned}
$$

where

$$
Q=-\frac{q^{2}}{M_{\alpha}^{2}}, \quad L_{\alpha}=\gamma+\ln \left(\frac{M_{\alpha}^{2}}{4 \pi \mu^{2}}\right), \quad d=4-2 \delta .
$$

The corresponding result for $J(1,1,1,1,0)$ is given by replacing $M_{\alpha}$ with $M_{\beta}$. We also find that

$$
\begin{aligned}
J(1,0,1,1,1)= & -\frac{1}{(4 \pi)^{4}}\left[-\frac{Q}{2 x} \frac{1}{\delta}+\frac{Q}{6(x-1) x^{2}}\left\{3 x^{2} L_{\alpha}-9 x^{2}-3 x L_{\alpha}-6 x \operatorname{Li}_{2}(1-b)\right.\right. \\
& \left.\left.+6 \operatorname{Li}_{2}(1-x)+\pi^{2} x+9 x-3 x \ln x-\pi^{2}\right\}\right]+\mathcal{O}\left(\frac{q^{4}}{M_{\alpha}^{4}}\right)
\end{aligned}
$$

and

$$
J(1,1,0,1,1)=K\left(q^{2}, M_{\alpha}\right) K\left(q^{2}, M_{\beta}\right)
$$

\footnotetext{
${ }^{4}$ In the notation of [30] corresponds to $T_{1234}\left(q^{2} ; M_{\alpha}^{2}, 0,0,0\right)$ of equation (96).
} 


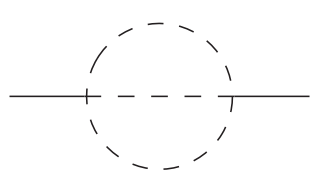

1. $J(0,1,1,1,0)$

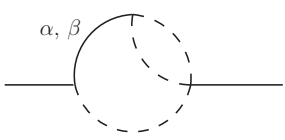

5. $J(0,1,1,1,1)$

$J(1,1,1,1,0)$

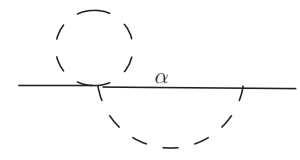

2. $J(0,1,1,0,1)$

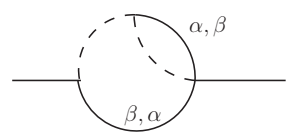

6. $J(1,0,1,1,1)$ $J(1,1,1,0,1)$

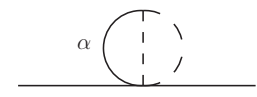

3. $J(0,0,1,1,1)$

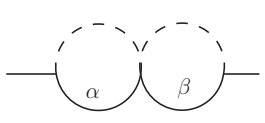

7. $J(1,1,0,1,1)$

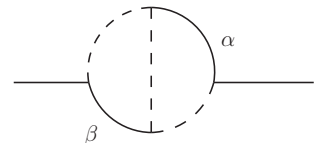

4. $J(-1,1,1,1,1)$

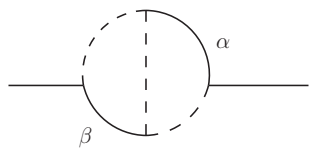

8. $J(1,1,1,1,1)$

Figure 14. The scalar topologies for $f_{1}$. The dashed lines now generically denote massless propagators, and so can correspond to both Higgs and lepton lines.

where

$$
K\left(q^{2}, M\right)=\frac{1}{\delta}-L_{M}+2+\ln \left(\frac{M^{2}}{M^{2}-\ell^{2}}\right)-\frac{M^{2}}{\ell^{2}} \ln \left(\frac{M^{2}}{M^{2}-\ell^{2}}\right)
$$

we also have

$$
J(-1,1,1,1,1)=I+\left(q^{2}-M_{\beta}^{2}\right) J(0,1,1,1,1)
$$

where

$$
I=\frac{M_{\alpha}^{2}}{(4 \pi)^{4}}\left\{\frac{1}{9} Q^{2}(4-3 \ln (Q))-\frac{1}{96} Q\left(24 L_{m}^{2}-84 L_{m}+10 \pi^{2}+105\right)\right\}+\mathcal{O}\left(\frac{q^{6}}{M_{\alpha}^{6}}\right) .
$$

Putting all this together, we find a total contribution to $I^{(4)}$ from $f_{1}$ of the the form

$$
f_{1}\left(q, M_{\alpha}, M_{\beta}\right)=\frac{\not q^{2}}{M_{\alpha} M_{\beta}} F_{[\alpha \beta]}^{(1)}+\cdots,
$$

where

$$
\begin{aligned}
F_{[\alpha \beta]}^{(1)}=\frac{1}{48(4 \pi)^{4} x^{2}}[ & 6 x^{4} \operatorname{Li}_{2}\left(\frac{x-1}{x}\right)-6 \operatorname{Li}_{2}(1-x)-\pi^{2} x^{4}+12 x^{3} \\
& \left.+3 x^{3} \ln (x)+3 x^{2} \ln (x)-12 x+3 x \ln (x)+\pi^{2}\right] .
\end{aligned}
$$

It has the asymptotic behaviour (characterised by $\xi=M_{\beta} / M_{\alpha}$ )

$$
\frac{F_{[\alpha \beta]}^{(1)}}{M_{\alpha} M_{\beta}} \simeq-\frac{1}{8} \frac{1}{(4 \pi)^{4}} \frac{1}{M_{\alpha}^{2}} \xi \ln \xi, \quad \xi \gg 1,
$$

as can be seen from the plot in figure 15 below.

For the other self-energy in figure 13, which contains no branch cuts, one could in principle carry out the same calculation. One begins by reducing the main diagram to a 


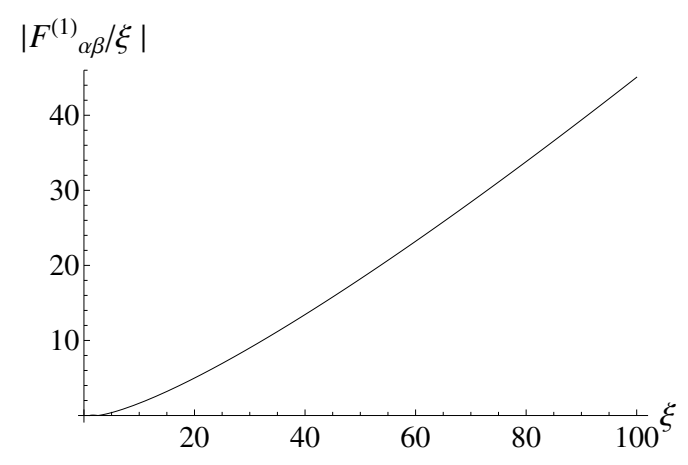

Figure 15. The hierarchy enhancement of loop effects from the $f_{1}$ term, with $M_{\alpha}$ fixed. As in figure 8 , we have taken out an overall factor $1 /(4 \pi)^{4}$.

sum of scalar integrals (figure 16), which gives a tensor decomposition into scalar integrals of the form

$$
\begin{aligned}
f_{2}=q \frac{M_{\alpha}}{M_{\beta}}[ & \frac{1}{2 q^{2}}(K(0,1,1,1,0)-K(0,1,1,0,1)-K(0,0,1,1,1)+K(-1,1,1,1,1)) \\
& +\left[\frac{2 M_{\beta}^{2}+M_{\alpha}^{2}}{2 q^{2}}-\frac{1}{2}\right] K(0,1,1,1,1)+\left[\frac{1}{2}-\frac{M_{\beta}^{2}}{q^{2}}\right] K(1,0,1,1,1)-\frac{1}{2} K(1,1,0,1,1) \\
& \left.-\frac{M_{\beta}^{2}}{q^{2}} K(1,1,1,0,1)+\frac{M_{\beta}^{2}}{q^{2}} K(1,1,1,1,0)+\left(-\frac{M_{\beta}^{2}}{2}+\frac{M_{\alpha}^{2} M_{\beta}^{2}}{q^{2}}\right) K(1,1,1,1,1)\right],
\end{aligned}
$$

where now

$$
\begin{aligned}
& K\left(\nu_{1}, \nu_{2}, \nu_{3}, \nu_{4}, \nu_{5}\right) \\
& \quad=\int \frac{d^{d} k_{1}}{(2 \pi)^{d}} \int \frac{d^{d} k_{2}}{(2 \pi)^{d}} \frac{1}{\left[k_{1}^{2}-M_{\beta}^{2}\right]^{\nu_{1}}\left[k_{2}^{2}-M_{\beta}^{2}\right]^{\nu_{2}}\left[\left(k_{1}+k_{2}\right)^{2}\right]^{\nu_{3}}\left[\left(k_{1}-q\right)^{2}\right]^{\nu_{4}}\left[\left(k_{2}+q\right)^{2}-M_{\alpha}^{2}\right]^{\nu_{5}}} .
\end{aligned}
$$

At this point, recall that we are mainly interested in diagrams which give the dominant asymptotic behaviour. By power counting in (4.29) and the diagrams in figure 16, one can see that only some of the diagrams (shown in bold) have the potential to give an asymptotic behaviour capable of competing with (4.28) or (4.12).

However, the asymptotic behaviour of these diagrams can be seen to be no stronger than in the previous calculations. The way to see this is to note that since none of them contains branch cuts, each one is analytic in $q^{2}$ and, as explained in [31], can be Taylor expanded before performing any momentum integrations, (see (2.6) therein). Each Taylor expansion can be written as a sum of 3-mass vacuum diagrams, each of which is given in terms of hypergeometric functions, as in (4.3) of [31]. One can then power count the masses $M_{\alpha}$ and $M_{\beta}$ in these formulae for each of the diagrams 4, 5, 6, 7 and 10. Carrying out this procedure, we found that in both limits $M_{\alpha} \gg M_{\beta}$ and $M_{\alpha} \ll M_{\beta}$, the asymptotic 


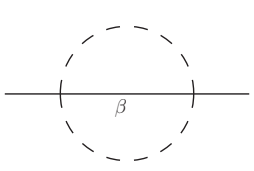

1. $K(0,1,1,1,0)$

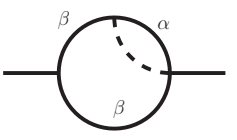

6. $K(1,0,1,1,1)$

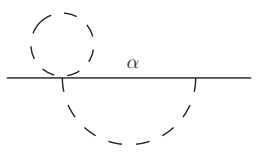

2. $K(0,1,1,0,1)$

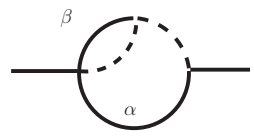

7. $K(1,1,1,0,1)$

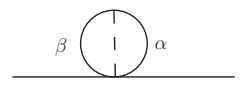

3. $K(0,0,1,1,1)$

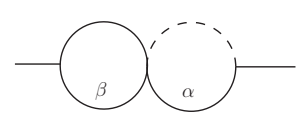

8. $K(1,1,0,1,1)$

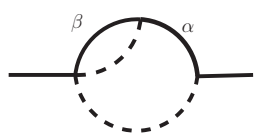

4. $K(-1,1,1,1,1)$

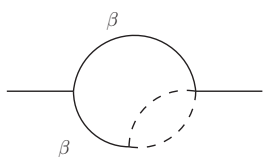

9. $K(1,1,1,1,0)$

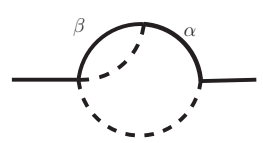

5. $K(0,1,1,1,1)$

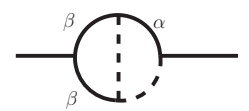

10. $K(1,1,1,1,1)$

Figure 16. Topologies for $f_{2}$.
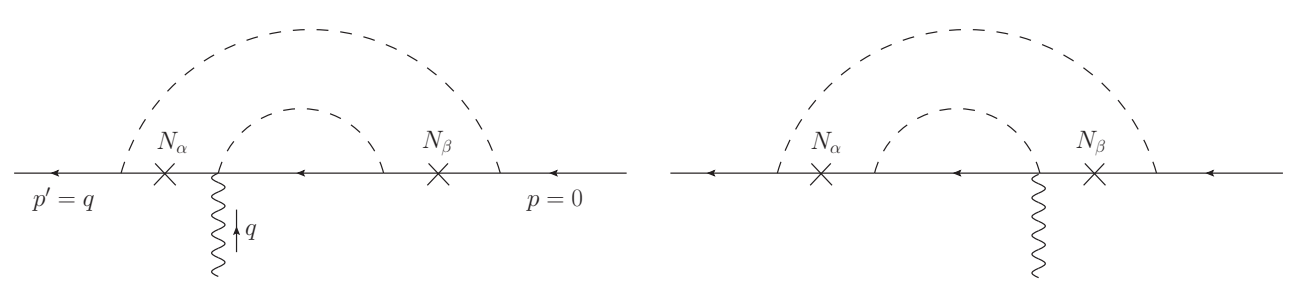

Figure 17. Contributions from Yukawa insertions.

behaviour is never stronger than in our previous calculations. As a result, we see that the dominant behaviour is essentially linear (up to an overall $\log \left(M_{\beta} / M_{\alpha}\right)$ factor).

\subsection{Yukawa couplings}

The contribution from the $h$ coupling to the Yukawa intercation in (3.10) is proportional to $(n-4)$, and so only contributes in diagrams which produce poles $1 /(n-4)$. The vertex correction diagram is UV finite, and so the only source of UV divergences comes from the propagator correction amplitude, via its one-loop sub-graph. In fact, for the purpose of calculating the antisymmetric quantity $I_{[\alpha \beta]}$, only two graviton insertions in the propagator correction diagram contribute. They are shown in figure 17.

Notice that we do not calculate the contributions from Yukawa insertions on the outermost vertices. The reason for this is that an insertion on the vertex of the outgoing lepton carries momentum $q$ in and straight back out again, so that the diagram has no $q$ dependence. Similarly, the insertion of $h$ on the vertex of the incoming lepton gives only a symmetric contribution to $I_{\alpha \beta}$. Moreover, in this case, the divergences in the sub-diagram are removed by renormalization counterterms in the corresponding one-loop diagrams. Hence, the only diagrams of interest are the two shown above. They give a contribution

$$
\begin{aligned}
I_{[\alpha \beta]} & =-\frac{1}{48} \frac{1+x}{(1-x)^{4}}\left[(1-x)\left(1+10 x+x^{2}\right)+6 x(1+x) \ln x\right] \\
& \simeq-\frac{1}{48}+O\left(\frac{\ln x}{x}\right),
\end{aligned}
$$

for large $x$. Clearly, this contribution is sub-dominant compared with our previous results. 


\subsection{Higgs couplings}

If we consider a conformally-coupled Higgs Lagrangian with $\zeta=1 / 6$, then from (3.10) the only $h$ couplings to the Higgs propagators are proportional to the mass $m_{\mathrm{H}}^{2}$. These contributions will therefore be highly suppressed relative to the insertions on the sterile neutrino propagators. For a non-conformal Higgs coupling, (3.10) shows there are further $h$ insertions proportional to $\left(\zeta-\frac{1}{6}\right) \partial^{2} h$. These have the potential to contribute in a similar way to the Yukawa terms discussed above, but power counting arguments suggest that they will again only give rise to sub-dominant contributions for large $x$.

\section{$5 \quad$ Leptogenesis and baryogenesis}

It is now clear how the mechanism of radiatively-induced gravitational leptogenesis emerges in this see-saw model. First, matter and antimatter propagate differently by virtue of the breaking of time-translational invariance by the gravitational background, together with $\mathrm{CP}$ violation from the phases of the complex Yukawa couplings. Both of these are radiativelyinduced features which arise at two loops, as discussed in section 1.1. This is manifest in the difference of the matter and antimatter self-energies, i.e. $\Sigma\left(x, x^{\prime}\right) \neq \Sigma^{c}\left(x, x^{\prime}\right)$. This bias in the dynamics of matter and antimatter is reflected in the particular operator (1.5), whose effective coupling constant depends on the imaginary part of the Yukawa couplings and on the sterile neutrino masses via the quantity $I_{\alpha \beta}$, which is determined by the twoloop self-energy diagrams for the light leptons. Naturally, these are the same diagrams (figures 5 and 7 ) which lead to distinct matter and antimatter propagation.

\subsection{Lepton asymmetry in a radiation-dominated FRW background}

We now show how the lepton asymmetry arises in the simplest possible model, where the background is a radiation-dominated FRW spacetime at temperature $T$. The coupling in (1.5) then acts as an effective chemical potential, so that in the thermal background of the hot early Universe, the equilibrium distributions of the light leptons and antileptons are different, giving rise to a net lepton number as shown in (1.5)-(1.7). We therefore find the lepton-to-photon ratio $Y_{L}$ in equilibrium is given by

$$
Y_{L} \simeq \frac{\pi^{2} \dot{R}}{2 \zeta(3) T} \sum_{\alpha, \beta, j, i} \frac{\operatorname{Im}\left[\lambda_{\beta i}^{\dagger} \lambda_{i \alpha} \lambda_{\beta j}^{\dagger} \lambda_{j \alpha}\right]}{18 M_{\alpha} M_{\beta}} I_{[\alpha, \beta]}(\xi),
$$

where we have used the photon density $n_{\gamma}=2 \zeta(3) T^{3} / \pi^{2}$.

We can trace the origin of each term in (5.1) back to the fundamental principles set out in the introduction. First, $\dot{R}$ arises due to the breaking of time-translation symmetry by the background geometry, the factor $I_{[\alpha, \beta]}(\xi) / M_{\alpha} M_{\beta}$ describes the dependence of the loops on the sterile neutrino mass hierachy, while $\operatorname{Im}\left[\lambda_{\beta i}^{\dagger} \lambda_{i \alpha} \lambda_{\beta j}^{\dagger} \lambda_{j \alpha}\right]$ arises from the breaking of $\mathrm{C}$ and $\mathrm{CP}$ symmetry.

At this point, we can also see why the diagrams containing charge-conserving sterile neutrino propagators do not contribute. (See section 2.1, especially expression (2.19) for 
the relevant self-energy diagram.) Since in this case the contribution from the vertices is $\left[\lambda_{\beta i}^{\dagger} \lambda_{i \alpha} \lambda_{\alpha j}^{\dagger} \lambda_{j \beta}\right]$, summing over all generations gives $n(L)-n\left(L^{c}\right) \propto \sum_{\alpha, \beta} \operatorname{Im}\left[\left(\lambda^{\dagger} \lambda\right)_{\beta \alpha}\left(\lambda^{\dagger} \lambda\right)_{\alpha \beta}\right]$ $\cdot J_{[\alpha \beta]}$, for some loop factor $J_{\alpha \beta}$. However, $\operatorname{Im}\left[\left(\lambda^{\dagger} \lambda\right)_{\beta \alpha}\left(\lambda^{\dagger} \lambda\right)_{\alpha \beta}\right]=\operatorname{Im}\left[\left|\left(\lambda^{\dagger} \lambda\right)_{\beta \alpha}\right|^{2}\right]=0$, and so the total lepton asymmetry from these diagrams is zero.

Now, the Ricci scalar in a FRW universe dominated by matter with equation of state parameter $w$ satisfies

$$
R=-(1-3 w) \frac{\rho}{M_{\mathrm{p}}^{2}}, \quad \dot{R}=\sqrt{3}(1-3 w)(1+w) \frac{\rho^{3 / 2}}{M_{\mathrm{p}}^{3}},
$$

where $M_{\mathrm{p}}$ is the reduced Planck mass, the expression for $\dot{R}$ following from the conservation and Friedmann equations. For the classically conformal invariant case of radiation dominance, $w=1 / 3$. At the quantum level, however, the energy-momentum tensor has a trace anomaly and the factor $(1-3 w)$ acquires a contribution from the beta functions characterising the particle content of the theory [11]. Below, we take $1-3 w \simeq 0.1$. Since for radiation, $\rho=\sigma T^{4}$ with $\sigma=\pi^{2} g_{*} / 30$, where $g_{*}$ counts the effective degrees of freedom, we find the time derivative of the curvature at temperature $T$ is

$$
\dot{R}=\sqrt{3} \sigma^{3 / 2}(1-3 w)(1+w) \frac{T^{6}}{M_{\mathrm{p}}^{3}} .
$$

Substituting back into (5.1) we find

$$
Y_{L} \simeq \frac{\sqrt{3} \pi^{2} \sigma^{3 / 2}(1-3 w)(1+w)}{36 \zeta(3)} \frac{T^{5}}{M_{\mathrm{p}}^{3}} \sum_{\alpha, \beta, j, i} \frac{\operatorname{Im}\left[\lambda_{\beta i}^{\dagger} \lambda_{i \alpha} \lambda_{\beta j}^{\dagger} \lambda_{j \alpha}\right]}{M_{\alpha} M_{\beta}} I_{[\alpha, \beta]}(\xi) .
$$

At this point, we can return to the discussion of the validity of the effective Lagrangian in section 3. Using the expression (5.2) for the curvature, the weak gravitational field and low-energy conditions can be re-expressed in terms of the temperature as

$$
\frac{T^{2}}{M_{1} M_{\mathrm{p}}} \ll 1, \quad \frac{T^{3}}{M_{1}^{2} M_{\mathrm{p}}} \ll 1,
$$

respectively, where we have taken the typical lepton energy as $E \sim T$ and $M_{1}$ as the sterile neutrino mass. These will clearly be satisfied in the region of interest, $T \sim M_{1}$ and $T \ll M_{\mathrm{p}}$. This means that the prediction (5.4) calculated from the effective Lagrangian is valid provided the temperature factor $\sim T^{5} / M_{1}^{2} M_{\mathrm{p}}^{3}$ in $Y_{L}$ is small, which is certainly the case observationally.

\subsection{Towards leptogenesis and baryogenesis}

Finally, we discuss briefly how this non-vanishing equilibrium lepton asymmetry may play a rôle in determining the baryon-to-photon ratio $\eta_{\mathrm{B}}$ of the Universe. We assume a standard leptogenesis scenario in which a lepton asymmetry established at relatively high temperature in a radiation-dominated FRW universe can subsequently be transformed into a baryon asymmetry by the conventional sphaleron mechanism [5] (see [7] for a summary) when the 


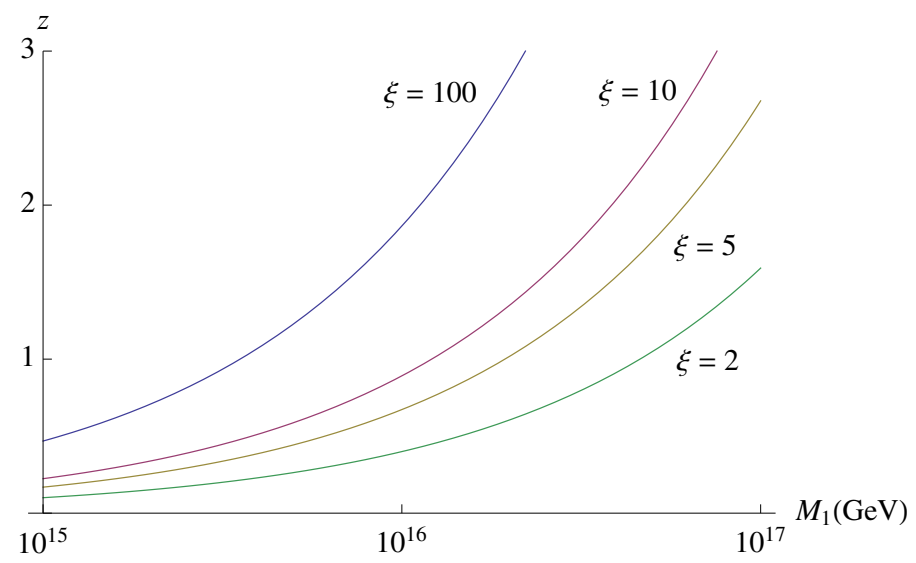

Figure 18. The curves show the values of $z=M_{1} / T$ and $M_{1}$ which give a lepton-to-photon ratio $Y_{L}=3 \times 10^{-8}$ in the observationally important range, for various values of the mass hierarchy parameter $\xi$.

temperature has dropped below the usual sphaleron scale $\sim 10^{12} \mathrm{GeV}$. From the observed value $\eta_{\mathrm{B}} \simeq 6 \times 10^{-10}$, we infer the corresponding ratio $Y_{L} \simeq 3 \times 10^{-8}$. ${ }^{5}$

To get an initial orientation on the relevant orders of magnitude, assume the sterile neutrino masses satisfy $M_{1} \ll M_{2} \ll M_{3}$. The expression (5.4) is then dominated by the loop diagrams with the largest mass hierarchy, i.e., those containing $M_{1}$ and $M_{3}$, which give $I_{[13]} \simeq \xi^{2} \log \xi^{2} /(4 \pi)^{4}$, where $\xi=M_{3} / M_{1}$. It is also convenient to introduce the standard parameter $z=M_{1} / T$. This gives our key result (5.4) in the form

$$
Y_{L} \simeq \alpha_{13}^{2} \sin \delta \frac{\xi \ln \xi^{2}}{z^{5}}\left(\frac{M_{1}}{M_{\mathrm{p}}}\right)^{3}\left[\frac{\sqrt{3} \sigma^{3 / 2} \pi^{2}(1-3 w)(1+w)}{36 \zeta(3)(4 \pi)^{2}}\right],
$$

where $\alpha_{13}=\left|\left(\lambda^{\dagger} \lambda\right)_{13}\right| /(4 \pi)$ is the appropriate coupling constant, and $\delta=\operatorname{Arg}\left[\left(\lambda^{\dagger} \lambda\right)_{13}^{2}\right]$ quantifies the size of $\mathrm{CP}$ violation. In the numerical estimate below, we take $\alpha_{13} \simeq 0.8$ and $\sin \delta \simeq 1$. Inserting these values, together with $(1-3 w)=0.1, g_{*}=106.75$ and $M_{\mathrm{p}}=2.4 \times 10^{18} \mathrm{GeV}$, we obtain the following expression for the equilibrium lepton-tophoton ratio at temperature $T$ :

$$
Y_{L} \simeq 4.4 \times 10^{-3} \frac{\xi \ln \xi^{2}}{z^{5}}\left(\frac{M_{1}}{M_{\mathrm{p}}}\right)^{3}
$$

In figure 18, we plot contours with $Y_{L}=3 \times 10^{-8}$ in the $z, M_{1}$ plane for different values of the hierarchy parameter $\xi$. This shows that the gravitationally-induced lepton asymmetry is indeed of the order of magnitude necessary to play a rôle in determining

${ }^{5}$ Here we have used the relation

$$
Y_{\mathrm{B}}=C_{\mathrm{sph}} Y_{B-L}=\frac{C_{\mathrm{sph}}}{1-C_{\mathrm{sph}}} Y_{L}
$$

with $C_{\mathrm{sph}}=28 / 79$ in the standard model, and included the standard factor $f=2387 / 86$ to account for the production of photons from the leptogenesis scale to CMB formation [8]. 
the observed baryon-to-photon ratio, with sterile neutrino masses and temperatures in the GUT range $\sim 10^{16} \mathrm{GeV}$.

In the see-saw model, which we have used here to illustrate the radiatively-induced gravitational mechanism, the sterile neutrino masses and hierarchy determine the light neutrino mass spectrum and these values correspond to neutrino masses of the order of a few $10^{-3} \mathrm{eV}$, putting them in the lower end of the range allowed by solar and atmospheric neutrino data [7]. Notice, however, that the fundamental gravitational effect, which gives rise to the chemical potential $\mu \sim \dot{R} / M_{1}^{2}$, is in general actually favoured by lower sterile neutrino masses. The relatively high mass parameters discussed above are being driven by the assumption that the relevant temperatures at which the lepton asymmetry freezes out are $z \gtrsim 1$.

If we consider the model in the context of the exit from inflation, then, as discussed in [11], this decoupling temperature must satisfy $T \lesssim T_{\mathrm{RH}} \lesssim V^{1 / 4}$, where $T_{\mathrm{RH}}$ is the reheat temperature and $V^{1 / 4}$ gives the inflationary scale. We would therefore require an inflationary scale of the order of the GUT scale, with the lepton asymmetry freezing out at high temperatures near the beginning of radiation dominance. This corresponds to a value of the tensor-to-scalar ratio $r$ (characterising the strength of the gravitational waves produced by inflation) to be close to the current upper bound $r_{\max }=0.07$ [34] .

The simplest scenario, implicit in the discussion above, is that the lepton asymmetry freezes out at its equilibrium value when the reaction rates for the lepton-number violating reactions, which maintain the leptons and antileptons in thermal equilibrium with the asymmetry (5.4), fall below the Hubble expansion parameter. Of course, this is a very crude first approximation. In general, finding the physically realised lepton asymmetry in this model will depend on a detailed dynamical analysis of all the simultaneous reactions and decays taking place around the critical scale $T \simeq M_{1}$. This would involve a full treatment of the coupled Boltzmann equations, taking into account initial abundances, inverse decays, and $\Delta L=1$ and $\Delta L=2$ scattering rates. This is essential to determine how closely the actual lepton asymmetry is tracking its equilibrium value at the point of freeze-out. We also need to include near-resonant production of the sterile neutrinos and their out-of-equilibrium decays, since in this model the original leptogenesis mechanism described in section 1.1 is simultaneously active.

This complete analysis of the kinetic theory of the model in its cosmological setting is currently under investigation and will be presented elsewhere. Here, our motivation has been simply to demonstrate that the new mechanism of radiatively-induced gravitational leptogenesis can produce asymmetries of the required order of magnitude to account for, or certainly play an important rôle in determining, the observed baryon-to-photon ratio in the Universe.

\section{Outlook}

In this paper, we have presented a new mechanism for leptogenesis and baryogenesis in which the matter-antimatter asymmetry is generated by gravitational couplings induced by quantum loop effects in curved spacetime. In this mechanism, the Sakharov conditions 
are realised as follows. The first occurs in the usual way for leptogenesis through lepton number violating reactions mediated by heavy BSM particles. $\mathrm{C}$ and $\mathrm{CP}$ violation can arise from complex phases in the coupling of the light leptons to these heavy states. The time dependence necessary, in the spirit of the third Sakharov condition, for generating a matterantimatter asymmetry arises not by the traditional mechanism of out-of-equilibrium decays of the BSM particles, but through the direct CP and SEP-violating coupling of quantum loops to the time-dependent gravitational field in the expanding Universe. In this scenario, the heavy BSM states contribute only as virtual particles to the self-energy cloud screening the light leptons. The lepton number asymmetry is then transferred to a baryon asymmetry via the usual sphaleron mechanism.

Specifically, we showed in the context of the see-saw model how the virtual sterile neutrinos in the two-loop self-energy contributions to the light lepton propagators allow the leptons to become sensitive to the time-dependent dynamics of the gravitational background and to the $\mathrm{C}$ and $\mathrm{CP}$ violation in the BSM Lagrangian. This curvature coupling breaks the strong equivalence principle and allows particles to propagate differently; the sensitivity to $\mathrm{CP}$ violation then allows a distinction between the dispersion relations for leptons and antileptons. This effect induces an effective chemical potential, which modifies the equilibrium distributions of leptons and antileptons and allows a lepton number asymmetry to be maintained in the thermal quasi-equilibrium characterising the early radiationdominated Universe. Remarkably for an intrinsically QFT effect in curved spacetime, this effect is sufficiently strong to play a rôle in determining the observed baryon-to-photon ratio. This is because, although the loop effects we have calculated are necessarily very small, they are the leading symmetry-breaking contribution to a quantity which would otherwise be zero by translation invariance. ${ }^{6}$

From our detailed calculations of the two-loop Feynman diagrams in the see-saw model, we made the important observation that that the size of the lepton (baryon) asymmetry grows linearly with the mass hierarchy $\xi=M_{\beta} / M_{\alpha}$ of the sterile neutrinos, and identified the class of diagrams giving rise to this enhancement. It would be interesting to develop a deeper understanding of this feature. For instance, is there an a priori reason why some diagrams such as the vertex correction (3) exhibit a hierarchy enhancement, whilst others such as the propagator-correction diagrams (1) and (2) do not? This would provide some insight into why we never observe anything stronger than linear hierarchy enhancement - clearly it would be of great interest for phenomenology if we found variations of the model in which the analogue of $I_{\alpha \beta}$ scales as $I_{\alpha \beta} \sim \xi^{4}$ or stronger. This could significantly extend the range of parameter values for which this type of see-saw model could generate a sizeable lepton asymmetry through our mechanism, for example reducing the scale $M_{1}$ of the lightest sterile neutrino.

This work suggests many areas for further investigation. The most immediate is to embed the mechanism into a detailed study of lepton number generation in the early radiationdominated Universe, taking into account the interplay between the conventional out-of-

\footnotetext{
${ }^{6}$ Another well-known example where curved spacetime QFT effects are important in cosmology is the study of quantum fluctuations in the inflationary phase of the Universe, which can be indirectly probed by CMB measurements.
} 
equilibrium decays of on-shell sterile neutrinos and our new mechanism of radiativelyinduced gravitational leptogenesis. This will involve a full analysis of the temperaturedependent, kinetic aspects of the evolution, including coupled Boltzmann equations, reaction rates and freeze-out temperatures as the Universe cools. An interesting question is to determine whether one mechanism dominates over the other for particular parameter ranges in the fundamental BSM theory.

It is important to emphasise, however, that the leptogenesis mechanism we are proposing is far more general than its realisation in the particular see-saw model presented in this paper. It will arise notably in generic BSM theories exhibiting $\mathrm{C}$ and $\mathrm{CP}$ violation at a high energy scale. The main condition is that since this is a gravitational leptogenesis mechanism, the matter-antimatter asymmetry must be generated at a sufficiently early time (around the GUT scale) that the curvature of the Universe is still strong enough to produce the observed baryon-to-photon ratio. Since this scale is also characteristic of the temperatures at the end of inflation, it will be interesting to look at scenarios where our mechanism is embedded into inflationary models. ${ }^{7}$ In principle, it can also be applied directly to the generation of a baryon asymmetry through a radiatively-generated gravitational coupling to the baryon number current.

On the theory side, the central idea underlying our mechanism is that in the presence of a time-varying gravitational field, matter and antimatter in a $\mathrm{C}$ and $\mathrm{CP}$-violating theory propagate differently at loop level. In this paper, we translated this fundamental observation into a mechanism for leptogenesis by first using the effective Lagrangian formalism to identify the relevant operator (1.5), then interpreting its coupling as a chemical potential which changes the dispersion relations and induces a difference in the equilibrium distributions for matter and antimatter. We showed that this operator arises naturally through radiative corrections in curved spacetime, without the need to appeal to an as yet unknown theory of quantum gravity. While this approach is justified in the early, GUT-scale Universe, where the traditional quasi-equilibrium approach to kinetic theory is a good approximation, from a theoretical perspective, we would like to develop a more fundamental analysis. An ideal strategy would be to describe the lepton (baryon) asymmetry directly from the self-energies $\Sigma\left(x, x^{\prime}\right)$, which should be treated within a real-time, non-equilibrium, curved spacetime framework to calculate the time evolution of the lepton (baryon) number. This would provide a theoretically rigorous, real-time formulation of radiatively-induced gravitational leptogenesis.

\section{Acknowledgments}

One of us (J.I. McD) would like to thank B. Garbrecht and V.A. Smirnov for helpful correspondence and advice. We are grateful to G. Aarts, D. De Boni and T. Hollowood for many useful conversations. This research is supported in part by STFC grants ST/K502376/1 and ST/L000369/1.

\footnotetext{
${ }^{7}$ See, for example, $[32,33]$ for models of leptogenesis in inflation.
} 


\section{A Techniques for evaluating the self-energy diagrams}

In this appendix, we demonstrate some of the techniques used for evaluating the Feynman diagrams in section 4. For instance, in the case of diagram (1), after inserting the subdiagram into the remaining momentum integral, we have

$$
f^{(1)}=\frac{M_{\alpha}}{2(4 \pi)^{2}} \int \frac{d^{d} k}{(2 \pi)^{d}} \frac{\left[(\not k+\not q) \not k+M_{\alpha}^{2}\right] \not k}{k^{2}\left[(k+q)^{2}-M_{\alpha}^{2}\right]\left[k^{2}-M_{\alpha}^{2}\right]\left[k^{2}-M_{\beta}^{2}\right]}\left(1-\frac{1}{2} \ln \left(\frac{-k^{2}}{\mu^{2}}\right)\right) .
$$

The constant term from $\Sigma(k)$ can be evaluated in the usual way, by manipulation of the numerator and introduction of Feynman parameters. The logarithmic term, however, is more subtle, but can be dealt with by noting we can write

$$
\ln \left(\frac{-k^{2}}{\mu^{2}}\right)=\lim _{s \rightarrow 0} \frac{d}{d s}\left[\frac{\left(-\mu^{2}\right)^{s}}{\left[k^{2}\right]^{s}}\right] .
$$

This trick can be employed for any other logarithmic term generated by a sub-diagram. This reduces the calculation to the evaluation of a Feynman integral containing a denominator factor raised to an arbitrary power $s$, viz.

$$
I(s) \equiv \frac{M_{\alpha}}{2(4 \pi)^{2}} \int \frac{d^{d} k}{(2 \pi)^{d}} \frac{\left.[(\not k+\not)) k+M_{\alpha}^{2}\right] \not k}{k^{2}\left[(k+q)^{2}-M_{\alpha}^{2}\right]\left[k^{2}-M_{\alpha}^{2}\right]\left[k^{2}-M_{\beta}^{2}\right]\left[k^{2}\right]^{s}},
$$

which can then be differentiated with respect to $s$ after performing the momentum integration. First, we note that the numerator can be rewritten as

$$
\left.(\not k+\not)) \not k+M_{\alpha}^{2}=(\not k+\not q)\left(k^{2}-M_{\alpha}^{2}\right)+(2 \not k+\not)\right) M_{\alpha}^{2},
$$

allowing us to cancel the numerator factor $\left(k^{2}-M_{\alpha}^{2}\right)$ against a denominator, so that $I(s)$ splits into two simpler integrals. For instance, the second term gives a contribution

$$
I(s)=\int \frac{d^{d} k}{(2 \pi)^{d}} \frac{(2 \not k+\not q)\left(-\mu^{2}\right)^{s}}{\left[k^{2}\right]\left[(k+q)^{2}-M_{\alpha}^{2}\right]\left[k^{2}-M_{\alpha}^{2}\right]\left[k^{2}-M_{\beta}^{2}\right]\left[k^{2}\right]^{s}},
$$

from which we need only the $O(s)$ term in accordance with (A.2). After introducing Feynman parameters, we get

$$
I(s)=\frac{\not}{(4 \pi)^{2}}\left(\frac{\mu^{2}}{M_{\alpha}^{2}}\right)^{s} \frac{s}{M_{\alpha}^{2}} \int_{0}^{1} d y \int_{0}^{1-y} d w \int_{0}^{1-y-w} d z \frac{w^{s-1}(y-1)}{[Q y(1-y)+y+z b]^{1+s}},
$$

where

$$
Q=-q^{2} / M_{\alpha}^{2}, \quad b=M_{\beta}^{2} / M_{\alpha}^{2} .
$$

Performing the $z$ and then $w$ integrals gives an answer in terms of incomplete Beta functions:

$$
\begin{gathered}
I(s)=\frac{\not d}{(4 \pi)^{2}}\left(\frac{\mu^{2}}{M_{\alpha}^{2}}\right)^{s} \frac{1}{M_{\beta}^{2}} \int_{0}^{1} d y(1-y)\left\{\frac{1}{b^{s}} B\left[\frac{b(1-y)}{Q y(1-y)+y+z(1-y-w)}, s, 1-s\right]\right. \\
\left.-\frac{1}{s} \frac{(1-y)^{s}}{[Q y(1-y)+y]^{s}}\right\} .
\end{gathered}
$$


Using the Taylor series for the incomplete beta function, we find the $s$ expansion gives

$$
\mathrm{B}[x, s, 1-s]=\frac{1}{s}+\ln (x)+s\left[\frac{1}{2} \ln ^{2}(x)+\operatorname{Li}_{2}(x)\right]+O\left(s^{2}\right) .
$$

Notice that poles in $s$ cancel between the Beta function and the second term in $\{\ldots\}$. Taking the term linear in $s$ gives

$$
\begin{aligned}
\lim _{s \rightarrow 0} \frac{d I(s)}{d s}=\frac{\not d}{(4 \pi)^{2}} \frac{1}{M_{\beta}^{2}} \int_{0}^{1} d y(1-y)\left\{\frac{1}{2}\right. & \ln ^{2}\left(\frac{\mu^{2}}{M_{\beta}^{2}}\right)+\ln \left(\frac{(1-y) b}{A}\right) \ln \left(L_{\beta}\right) \\
& +\frac{1}{2} \ln ^{2}\left(\frac{(1-y) b}{A}\right)+\operatorname{Li}_{2}\left(\frac{(1-y) b}{A}\right) \\
& \left.-\frac{1}{2} \ln ^{2}\left(\frac{(1-y)}{Q y(1-y)+y}\right)\right\},
\end{aligned}
$$

where

$$
A=Q y(1-y)+y+(1-y) b .
$$

A full expression for the remaining $y$ integration is too lengthy to write down here. However, each of the terms in the integrand is analytic in $q$ for all $y$ in the integration range, provided $-q^{2} \ll M_{\alpha}^{2}, M_{\beta}^{2}$, and so the integrand can be analytically expanded in powers of $q^{2} / M_{\alpha}^{2}$ prior to performing the $y$ integral. This is a reflection of the fact this diagram contains no branch cuts, i.e., no zero-mass thresholds, and thus has no $\log \left(-q^{2} / M_{\alpha}^{2}\right)$ terms. Expanding in $-q^{2} / M_{\alpha}^{2}$, and then performing the $y$ integral, we find an answer of the form

$$
\lim _{s \rightarrow 0} \frac{d I(s)}{d s}=\frac{q}{(4 \pi)^{2}} \frac{1}{M_{\beta}^{2}} \sum_{n} C_{n}\left(\frac{-q^{2}}{M_{\alpha}^{2}}\right)^{n},
$$

where for our purposes the relevant coefficient is

$$
C_{1}=-\frac{(b((b-6) b+3)+6 b \ln (b)+2) \ln \left(\frac{\mu^{2}}{M_{\alpha}^{2}}\right)-3 b \ln ^{2}(b)+3(b-1)^{2}}{6(b-1)^{4}}-\frac{2 \ln \left(\frac{\mu^{2}}{M_{\alpha}^{2}}\right)+3}{6} .
$$

We can repeat this exercise for the other integrals contributing to diagram (1) to arrive at the answer quoted in the text in (4.4).

Similar techniques are used to evaluate the other self-energy diagrams, with the results quoted in section 4 .

Open Access. This article is distributed under the terms of the Creative Commons Attribution License (CC-BY 4.0), which permits any use, distribution and reproduction in any medium, provided the original author(s) and source are credited.

\section{References}

[1] J.I. McDonald and G.M. Shore, Radiatively-induced gravitational leptogenesis, Phys. Lett. B 751 (2015) 469 [arXiv:1508.04119] [InSPIRE].

[2] A. Sakharov, Violation of CP invariance, $C$ asymmetry, and baryon asymmetry of the universe, Sov. Phys. Usp. 34 (1991) 392 [Pisma Zh. Eksp. Teor. Fiz. 5 (1967) 32] [JETP Lett. 5 (1967) 24] [Usp. Fiz. Nauk 161 (1991) 61] [INSPIRE]. 
[3] M. Fukugita and T. Yanagida, Baryogenesis Without Grand Unification, Phys. Lett. B 174 (1986) 45 [INSPIRE].

[4] F.R. Klinkhamer and N.S. Manton, A Saddle Point Solution in the Weinberg-Salam Theory, Phys. Rev. D 30 (1984) 2212 [inSPIRE].

[5] V.A. Kuzmin, V.A. Rubakov and M.E. Shaposhnikov, On the Anomalous Electroweak Baryon Number Nonconservation in the Early Universe, Phys. Lett. B 155 (1985) 36 [INSPIRE].

[6] E.W. Kolb and M.S. Turner, The Early Universe, Addison Wesley (1990) [Front. Phys. 69 (1990) 1] [INSPIRE].

[7] W. Buchmüller, Baryo-and Leptogenesis (Brief Summary), in proceedings of the ICTP Summer School on Astroparticle Physics and Cosmology, Trieste, Italy, June 17-July 5 2002, G. Dvali, A. Perez-Lorenzana, G. Senjanovic, G. Thompson and F. Vissani eds., ICTP, Trieste Italy (2003), pp. 41-75 [INSPIRE].

[8] W. Buchmüller, P. Di Bari and M. Plümacher, Leptogenesis for pedestrians, Annals Phys. 315 (2005) 305 [hep-ph/0401240] [INSPIRE].

[9] E.W. Kolb and S. Wolfram, Baryon Number Generation in the Early Universe, Nucl. Phys. B 172 (1980) 224 [Erratum ibid. B 195 (1982) 542] [INSPIRE].

[10] A.G. Cohen and D.B. Kaplan, Thermodynamic Generation of the Baryon Asymmetry, Phys. Lett. B 199 (1987) 251 [INSPIRE].

[11] H. Davoudiasl, R. Kitano, G.D. Kribs, H. Murayama and P.J. Steinhardt, Gravitational baryogenesis, Phys. Rev. Lett. 93 (2004) 201301 [hep-ph/0403019] [INSPIRE].

[12] G. Lambiase and S. Mohanty, Gravitational Leptogenesis, JCAP 12 (2007) 008 [astro-ph/0611905] [INSPIRE].

[13] G. Lambiase and S. Mohanty, Leptogenesis by curvature coupling of heavy neutrinos, Phys. Rev. D 84 (2011) 023509 [arXiv:1107.1213] [INSPIRE].

[14] G. Lambiase, S. Mohanty and A.R. Prasanna, Neutrino coupling to cosmological background: A review on gravitational Baryo/Leptogenesis, Int. J. Mod. Phys. D 22 (2013) 1330030 [arXiv: 1310.8459$]$ [INSPIRE].

[15] L. Pizza, Baryo-Leptogenesis induced by modified gravities in the primordial Universe, arXiv:1506.08321 [INSPIRE].

[16] J.I. McDonald and G.M. Shore, Gravitational leptogenesis, C, CP and strong equivalence, JHEP 02 (2015) 076 [arXiv: 1411.3669] [INSPIRE].

[17] R.F. Streater and A.S. Wightman, PCT, spin and statistics, and all that, Princeton University Press, Princeton U.S.A. (2000).

[18] I.T. Drummond and S.J. Hathrell, QED Vacuum Polarization in a Background Gravitational Field and Its Effect on the Velocity of Photons, Phys. Rev. D 22 (1980) 343 [INSPIRE].

[19] Y. Ohkuwa, Effect of a Background Gravitational Field on the Velocity of Neutrinos, Prog. Theor. Phys. 65 (1981) 1058 [INSPIRE].

[20] T.J. Hollowood and G.M. Shore, The Causal Structure of QED in Curved Spacetime: Analyticity and the Refractive Index, JHEP 12 (2008) 091 [arXiv:0806.1019] [INSPIRE].

[21] T.J. Hollowood, G.M. Shore and R.J. Stanley, The Refractive Index of Curved Spacetime II: QED, Penrose Limits and Black Holes, JHEP 08 (2009) 089 [arXiv:0905.0771] [InSPIRE]. 
[22] T.J. Hollowood and G.M. Shore, The Effect of Gravitational Tidal Forces on Renormalized Quantum Fields, JHEP 02 (2012) 120 [arXiv:1111.3174] [INSPIRE].

[23] F.A. Berends and R. Gastmans, Quantum Electrodynamical Corrections to Graviton-Matter Vertices, Annals Phys. 98 (1976) 225 [InSPIRE].

[24] J.F. Donoghue, B.R. Holstein, B. Garbrecht and T. Konstandin, Quantum corrections to the Reissner-Nordstrom and Kerr-Newman metrics, Phys. Lett. B 529 (2002) 132 [hep-th/0112237] [INSPIRE].

[25] N.E.J. Bjerrum-Bohr, J.F. Donoghue and B.R. Holstein, Quantum corrections to the Schwarzschild and Kerr metrics, Phys. Rev. D 68 (2003) 084005 [Erratum ibid. D 71 (2005) 069904] [hep-th/0211071] [INSPIRE].

[26] G. 't Hooft and M.J.G. Veltman, Scalar One Loop Integrals, Nucl. Phys. B 153 (1979) 365 [INSPIRE].

[27] D. Kreimer, The Master two loop two point function: The General case, Phys. Lett. B 273 (1991) 277 [INSPIRE].

[28] F.A. Berends, A.I. Davydychev, V.A. Smirnov and J.B. Tausk, Zero threshold expansion of two loop selfenergy diagrams, Nucl. Phys. B 439 (1995) 536 [hep-ph/9410232] [INSPIRE].

[29] V.A. Smirnov, Asymptotic expansions in momenta and masses and calculation of Feynman diagrams, Mod. Phys. Lett. A 10 (1995) 1485 [hep-th/9412063] [INSPIRE].

[30] R. Scharf and J.B. Tausk, Scalar two loop integrals for gauge boson selfenergy diagrams with a massless fermion loop, Nucl. Phys. B 412 (1994) 523 [INSPIRE].

[31] A.I. Davydychev and J.B. Tausk, Two loop selfenergy diagrams with different masses and the momentum expansion, Nucl. Phys. B 397 (1993) 123 [INSPIRE].

[32] S.H.S. Alexander, M.E. Peskin and M.M. Sheikh-Jabbari, Leptogenesis from gravity waves in models of inflation, Phys. Rev. Lett. 96 (2006) 081301 [hep-th/0403069] [INSPIRE].

[33] M. Bastero-Gil, A. Berera, R.O. Ramos and J.G. Rosa, Warm baryogenesis, Phys. Lett. B 712 (2012) 425 [arXiv: 1110.3971] [INSPIRE].

[34] BICEP2 and KeCK Array collaborations, P.A.R. Ade et al., Improved Constraints on Cosmology and Foregrounds from BICEP2 and Keck Array Cosmic Microwave Background Data with Inclusion of 95 GHz Band, Phys. Rev. Lett. 116 (2016) 031302 [arXiv: 1510.09217] [INSPIRE]. 